\title{
The MOPED framework: Object Recognition and Pose Estimation for Manipulation
}

\author{
Alvaro Collet $^{*} \quad$ Manuel Martinez ${ }^{\dagger} \quad$ Siddhartha S. Srinivasa ${ }^{\ddagger}$
}

\begin{abstract}
We present MOPED, a framework for Multiple Object Pose Estimation and Detection that seamlessly integrates single-image and multi-image object recognition and pose estimation in one optimized, robust, and scalable framework. We address two main challenges in computer vision for robotics: robust performance in complex scenes, and low latency for real-time operation.

We achieve robust performance with Iterative ClusteringEstimation (ICE), a novel algorithm that iteratively combines feature clustering with robust pose estimation. Feature clustering quickly partitions the scene and produces object hypotheses. The hypotheses are used to further refine the feature clusters, and the two steps iterate until convergence. ICE is easy to parallelize, and easily integrates single- and multi-camera object recognition and pose estimation. We also introduce a novel object hypothesis scoring function based on M-estimator theory, and a novel pose clustering algorithm that robustly handles recognition outliers.

We achieve scalability and low latency with an improved feature matching algorithm for large databases, a GPU/CPU hybrid architecture that exploits parallelism at all levels, and an optimized resource scheduler. We provide extensive experimental results demonstrating state-of-the-art performance in terms of recognition, scalability, and latency in real-world robotic applications.
\end{abstract}

\section{Introduction}

The task of estimating the pose of a rigid object model from a single image is a well studied problem in the literature. In the case of point-based features, this is known as the Perspective$n$-Point (PnP) problem (Fischler and Bolles, 1981), for which many solutions are available, both closed-form (Lepetit et al., 2008) and iterative (Dementhon and Davis, 1995). Assuming that enough perfect correspondences between 2D image features and 3D model features are known, one only needs to use the $\mathrm{PnP}$ solver of choice to obtain an estimation of an

${ }^{*}$ A. Collet is with The Robotics Institute, Carnegie Mellon University, 5000 Forbes Ave., Pittsburgh, PA - 15213, USA. \{acollet\}@cs.cmu .edu

${ }^{\dagger} \mathrm{M}$. Martinez is with The Robotics Institute, Carnegie Mellon University, 5000 Forbes Ave., Pittsburgh, PA - 15213, USA. \{manelm\}@cs.cmu .edu

${ }^{\ddagger}$ Siddhartha S. Srinivasa Intel Labs Pittsburgh 4720 Forbes Avenue, Suite 410, Pittsburgh, PA - 15213, USA. \{siddhartha.srinivasa\}@intel.com

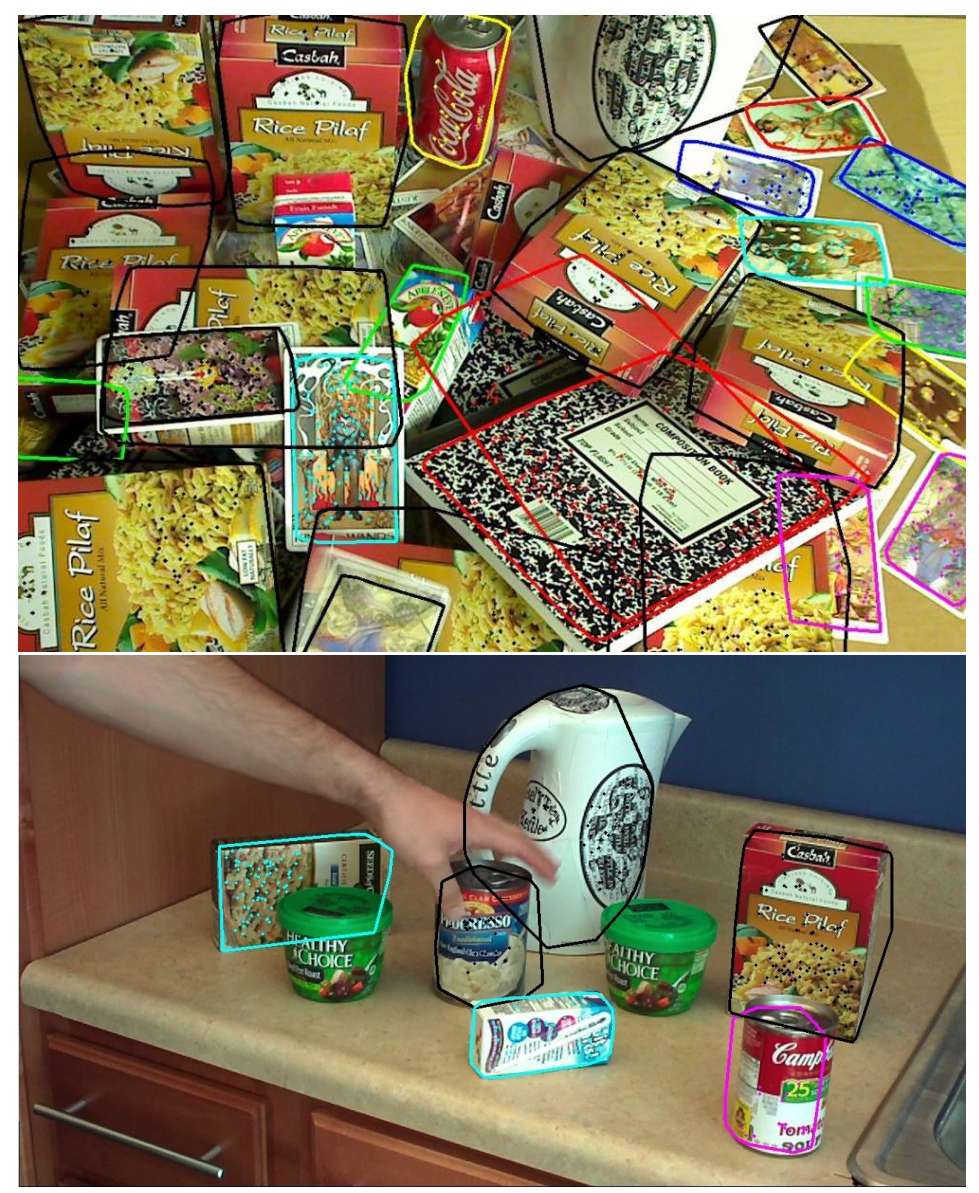

Figure 1: Recognition of real-world scenes. (Top) Highcomplexity scene. MOPED finds 27 objects, including partiallyoccluded, repeated and non-planar objects. Using a database of 91 models and an image resolution of $1600 \times 1200$, MOPED processes this image in 2.1 seconds. (Bottom) Medium complexity scene. MOPED processes this $640 \times 360$ image in $87 \mathrm{~ms}$ and finds all known objects (The undetected green soup can is not in the database).

object's pose. When noisy measurements are considered, nonlinear least-squares minimization techniques (e.g. LevenbergMarquardt (Marquardt, 1963)) often provide better pose estimates. Given that such techniques require good initialization, a closed-form PnP solver is often used to initialize the non-linear minimizer.

The very related task of recognizing a single object and de- 
termining its pose from a single image requires solving two sub-problems: finding enough correct correspondences between image features and model features, and estimating the model pose that best agrees with that set of correspondences. Even with highly discriminative locally invariant features, such as SIFT (Lowe, 2004) or SURF (Bay et al., 2008), mismatched correspondences are inevitable, forcing us to utilize robust estimation techniques such as M-estimators or RANSAC (Fischler and Bolles, 1981) in most modern object recognition systems. A comprehensive overview of model-based 3D object recognition/tracking techniques is available at Lepetit and Fua (2005).

The problem of model-based 3D object recognition is mature, with a vast literature behind, but it is far from solved in its most general form. Two problems greatly influence the performance of any recognition algorithm.

The first problem is scene complexity. This can arise due to feature count, with both extremes (too many features, or too few) significantly decreasing recognition rate. Related is the issue of repeated objects: the matching ambiguity introduced by repeated instances of an object presents an enormous challenge for robust estimators, as the matched features might belong to different object instances despite being correct. Solutions such as grouping (Lowe, 1987), interpretation trees (Grimson, 1991) or image space clustering (Collet et al., 2009) are often used, but false positives often arise from algorithms not being able to handle unexpected scene complexity. Fig. 1 shows an example of an image of high complexity and multiple repeated objects correctly processed by MOPED.

The second problem is that of scalability and system latency. In systems that operate online, a trade-off between recognition performance and latency must be reached, depending on the requirements for each specific task. In robotics, the reaction time of robots operating in dynamic environments is often limited by the latency of their perception (e.g. (Srinivasa et al., 2010, WillowGarage, 2008)). Increasing the volume of input data to process (e.g. increasing the image resolution, using multiple cameras) usually results in a severe penalty in terms of processing time. Yet, with cameras getting better, cheaper, and smaller, multiple high resolution views of a scene are often easily available. For example, our robot HERB has, at various times, been outfitted with cameras on its shoulder, in the palm, on its ankle-high laser, as well as with a stereo pair. Multiple views of a scene are often desirable, because they provide depth estimation, robustness against line-of-sight occlusions, and an increased effective field of view. Fig. 2 shows MOPED applied on a set of three images for grasping. Also, the higher resolution can potentially improve the recognition of complicated objects and the accuracy of pose estimation algorithms, but often at a steep penalty cost, as the extra resolution often causes an increase in the number of false positives as well as severe degradation in terms of latency and throughput.

In this paper, we address these two problems in model-based 3D object recognition through multiple novel contributions, both algorithmic and architectural. We provide a scalable framework for object recognition specifically designed to address increased scene complexity, limit false positives, and uti-

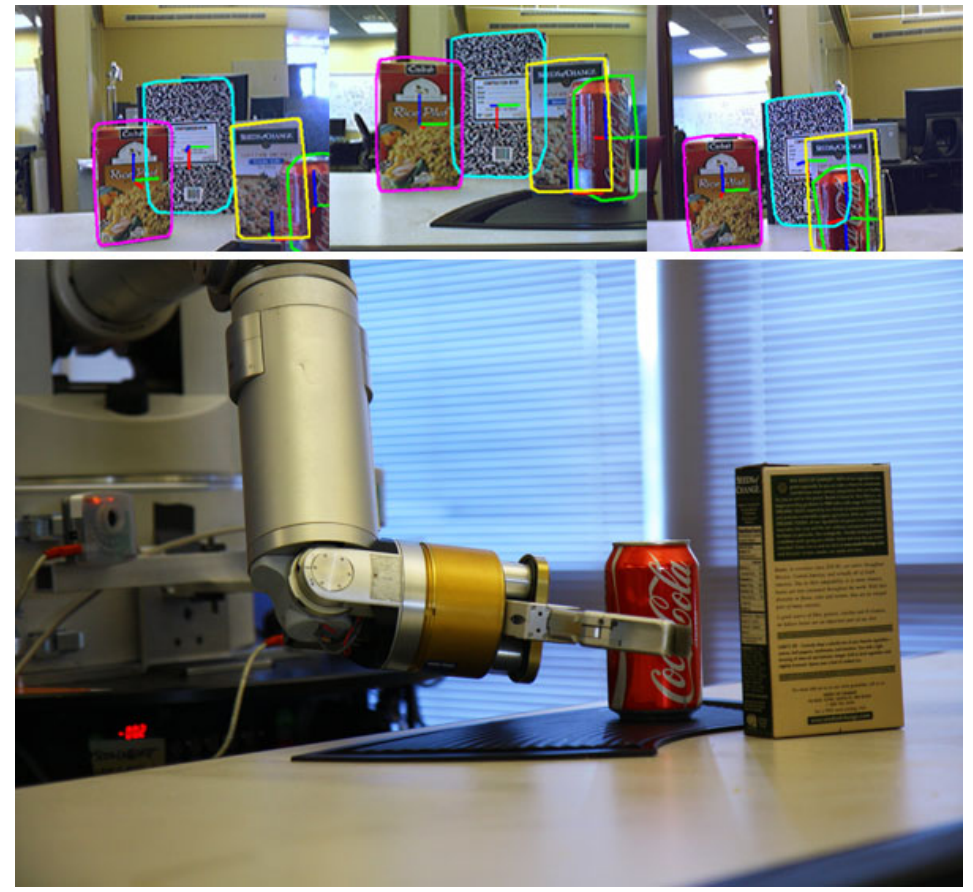

Figure 2: Object grasping in a cluttered scene using MOPED. (Top) Scene observed by a set of three cameras. (Bottom) Our robotic platform HERB (Srinivasa et al., 2010) in the process of grasping an object, using only the pose information from MOPED.

lize all computing resources to provide low latency processing for one or multiple simultaneous high-resolution images. The Iterative Clustering-Estimation (ICE) algorithm is our most important contribution to handle scenes with high complexity and keep latency low. In essence, ICE jointly solves the correspondence and pose estimation problems through an iterative procedure. ICE estimates groups of features that are likely to belong to the same object through clustering, and then searches for object hypotheses within each of the groups. Each hypothesis found is used to refine the feature groups that are likely to belong to the same object, which in turn helps finding more accurate hypotheses. The iteration of this procedure focuses the object search only in the regions with potential objects, avoiding the waste of processing power in unlikely regions. In addition, ICE allows for an easy parallelization and the integration of multiple cameras in the same joint optimization.

Another important contribution of this paper is a robust metric to rank object hypotheses based on M-estimator theory. A common metric used in model-based $3 \mathrm{D}$ object recognition is the sum of reprojection errors. However, this metric prioritizes objects that have been detected with the least amount of information, since each additional recognized object feature is bound to increase the overall error. Instead, we propose a quality metric that encourages objects to have as most correspondences as possible, thus achieving more stable estimated poses. This metric is relied upon in the clustering iterations within ICE, and is specially useful when coupled with our novel pose clustering algorithm. The key insight behind our pose clustering algorithm 
- called Projection Clustering - is that our object hypotheses have been detected from camera data, which might be noisy, ambiguous and/or contain matching outliers. Therefore, instead of using a regular clustering technique in pose space (using e.g. Mean Shift (Cheng, 1995) or Hough Transforms (Olson, 1997)), we evaluate each type of outlier and propose a solution that handles incorrect object hypotheses and effectively merges their information with those that are most likely to be correct.

This work also tackles the issues of scalability, throughput and latency, which are vital for real-time robotics applications. ICE enables easy parallelism in the object recognition process. We also introduce an improved feature matching algorithm for large databases that balances matching accuracy and logarithmic complexity. Our GPU/CPU hybrid architecture exploits parallelism at all levels. MOPED is optimized for bandwidth and cache management and SIMD instructions. Components like feature extraction and matching have been implemented on a GPU. Furthermore, a novel scheduling scheme enables the efficient use of symmetric multiprocessing(SMP) architectures, utilizing all available cores on modern multi-core CPUs.

Our contributions are validated through extensive experimental results demonstrating state-of-the-art performance in terms of recognition, pose estimation accuracy, scalability, throughput and latency. Five benchmarks and a total of over 6000 images are used to stress-test every component of MOPED. The different benchmarks are executed in a database of 91 objects, and contain images with up to 400 simultaneous objects, high-definition video footage, and a multi-camera setup.

Preliminary versions of this work have been published at Collet et al. (2009), Collet and Srinivasa (2010), Martinez et al. (2010). Additional information, videos, and the full source code of MOPED are available online at http://personalrobotics . intel-research.net/projects/moped.

\section{Problem formulation}

The goal of MOPED is the recognition of objects from images given a database of object models, and the estimation of the pose of each recognized object. In this section, we formalize these inputs and outputs and introduce the terminology we use throughout the paper.

\subsection{Input: images}

The input to MOPED is a set $\mathbf{I}$ of $M$ images

$$
\mathbf{I}=\left\{I_{1}, \ldots, I_{m}, \ldots, I_{M}\right\} \quad I_{m}=\left\{K_{m}, T_{m}, \mathbf{g}_{m}\right\} .
$$

In the general case, each image is captured with a different calibrated camera. Therefore, each image $I_{m}$ is defined by a $3 \times 3$ matrix of intrinsic camera parameters $K_{m}$, a $4 \times 4$ matrix of extrinsic camera parameters $T_{m}$ with respect to a known world reference frame, and a matrix of pixel values $\mathbf{g}_{m}$.

MOPED is agnostic to the number of images $M$. In other words, it is equally valid in both an extrinsically calibrated multi-camera setup, and in the simplified case of a single image
$(M=1)$ and a camera-centric world $\left(T_{1}=\mathcal{I}_{4}\right.$, where $\mathcal{I}_{4}$ is a $4 \times 4$ identity matrix).

\subsection{Input: object models}

Each object to be recognized by MOPED first goes through an offline learning stage, in which a sparse 3D model of the object is created. First, a set of images is taken with the object in various poses. Reliable local descriptors are extracted from natural features using SIFT(Lowe, 2004), which have proven to be one of the most distinctive and robust local descriptors across a wide range of transformations (Mikolajczyk and Schmid, 2005). Alternative descriptors (e.g. SURF(Bay et al., 2008), ferns(Ozuysal et al., 2010)) can also be used. Using structure from motion (Szeliski and Kang, 1994) on the matched SIFT keypoints, we merge the information from each training image into a sparse 3D model. Each 3D point is linked to a descriptor that is produced from clustering individual matched descriptors in different views. Finally, proper alignment and scale for each model are computed to match the real object dimensions and define an appropriate coordinate frame, which for simplicity is defined at the object's center.

Let $\mathbf{O}$ be a set of object models. Each object model is defined by its object identity $o$ and a set of features $\mathbf{F}_{o}$

$$
O=\left\{o, \mathbf{F}_{o}\right\} \quad \mathbf{F}_{o}=\left\{F_{1 ; o}, \ldots, F_{i ; o}, \ldots, F_{N ; o}\right\} .
$$

Each feature is represented by a 3D point location $P=$ $[X, Y, Z]^{T}$ in the object's coordinate frame and a feature descriptor $D$, whose dimensionality depends on the type of descriptor used, e.g. $k=128$ if using SIFT or $k=64$ if using SURF. That is,

$$
F_{i ; o}=\left\{P_{i ; o}, D_{i ; o}\right\} \quad P_{i ; o} \in \mathbb{R}^{3}, D_{i ; o} \in \mathbb{R}^{k} .
$$

The union of all features from all objects in $\mathbf{O}$ is defined as $\mathbf{F}=\bigcup_{o \in \mathbf{O}} \mathbf{F}_{o}$.

\subsection{Output: recognized objects}

The output of MOPED is a set of object hypotheses $\mathbf{H}$. Each object hypothesis $H_{h}=\left\{o, T_{h}\right\}$ is represented by an object identity $o$ and a $4 \times 4$ matrix $T_{h}$ that corresponds to the pose of the object with respect to the world reference frame.

\section{Iterative Clustering-Estimation}

The task of recognizing objects from local features in images requires solving two sub-problems: the correspondence problem and the pose estimation problem. The correspondence problem refers to the accurate matching of image features to features that belong to a particular object. The pose estimation problem refers to the generation of object poses that are geometrically consistent with the found correspondences.

The inevitable presence of mismatched correspondences forces us to utilize robust estimation techniques, such as Mestimators or RANSAC (Fischler and Bolles, 1981). In the 
(b)

(a)

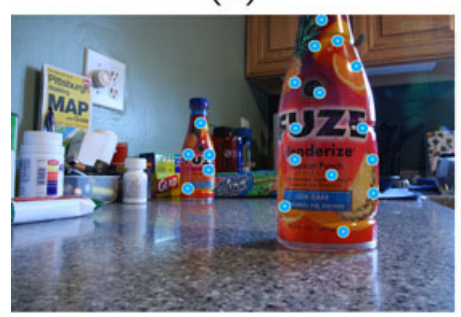

(c)

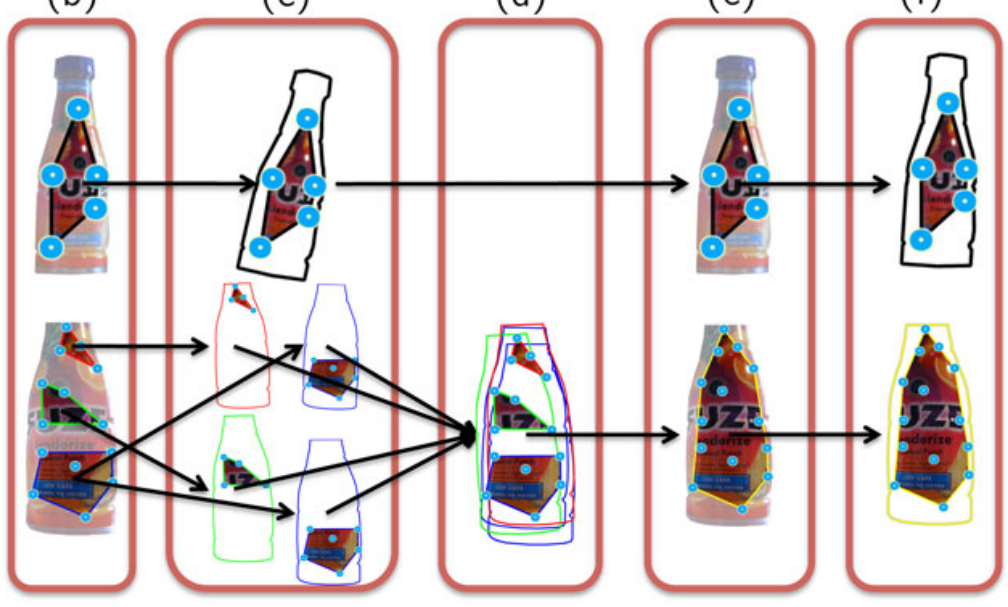

(g)

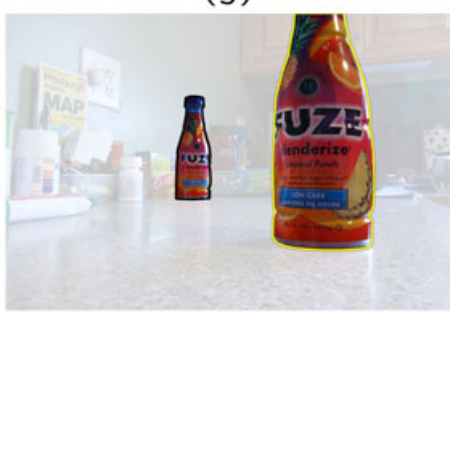

Figure 3: Illustration of two ICE iterations. Colored outlines represent estimated poses. (a) Feature extraction and matching. (b) Feature clustering. (c) Hypothesis generation. (d-e) Cluster clustering. (f) Pose refinement. (g) Final result.

presence of repeated objects in a scene, the correspondence problem cannot be solved in isolation, as even perfect image-tomodel correspondences need to be linked to a particular object instance. Robust estimation techniques often fail as well in the presence of this increased complexity. Solutions such as grouping (Lowe, 1987), interpretation trees (Grimson, 1991) or image space clustering (Collet et al., 2009) alleviate the problem of repeated objects by reducing the search space for object hypotheses.

The Iterative Clustering-Estimation (ICE) algorithm at the heart of MOPED aims to jointly solve the correspondence and pose estimation problems in a principled way. Given initial image-to-model correspondences, ICE iteratively executes clustering and pose estimation to progressively refine which features belong to each object instance, and to compute the object poses that best fit each object instance. The algorithm is illustrated in Fig. 3.

Given a scene with a set of matched features, (Fig. 3.a), the Clustering step generates groups of image features that are likely to belong to a single object instance (Fig. 3.b). If prior object pose hypotheses are available, features consistent with each object hypothesis are used to initialize distinct clusters. Numerous object hypotheses are generated for each cluster (Fig. 3.c). Then, object hypotheses are merged together if their poses are similar (Fig. 3.d), thus uniting their feature clusters into larger clusters that potentially contain all information about a single object instance (Fig. 3.e). With multiple images, the use of a common reference frame allows us to link object hypotheses recognized in different images, and thus create multi-image feature clusters. If prior object pose hypotheses are not available (i.e. at the first iteration of ICE), we use the density of local features matched to an object model as a prior, with the intuition that groups of features spatially close together are more likely to belong to the same object instance than features spread across all images. Thus, we initialize ICE with clusters of features in image space $(x, y)$, as seen in Fig. 3.b.
The Estimation step computes object hypotheses given clusters of features (as shown in Fig. 3.c and Fig. 3.f). Each cluster can potentially generate one or multiple object hypotheses, and also contain outliers that cannot be used for any hypothesis. A common approach for hypothesis generation is the use of RANSAC along with a pose estimation algorithm, although other approaches are equally valid. In RANSAC, we choose subsets of features at random within the cluster, then hypothesize an object pose that best fits the subset of features, and finally check how many features in the cluster are consistent with the pose hypothesis. This process is repeated multiple times and a set of object hypotheses is generated for each cluster. The advantage of restricting the search space to that of feature clusters is the higher likelihood that features from only one object instance are present, or at most a very limited number of them. This process can be performed regardless of whether the features belong to one or multiple images.

The set of hypotheses from the Estimation step are then utilized to further refine the membership of each feature to each cluster (Fig. 3.d-e). The whole process is iterated until convergence, which is reached when no features change their membership in a Clustering step (Fig. 3.f).

In practice, ICE requires very few iterations until convergence, usually as little as 2 for setups with one or a few simultaneous images. Parallelization is easy, since the initial steps are independent for each cluster in each image and object type. Therefore, large sets of images can be potentially integrated into ICE with very little impact on overall system latency. Two ICE iterations are required for increased robustness and speed in setups ranging from one to a few simultaneous images, while further iterations of ICE might potentially be necessary if tens or hundreds of simultaneous images are to be processed. 


\subsection{ICE as Expectation-Maximization}

It is interesting to note the conceptual similarity between ICE and the well-known Expectation-Maximization (EM) (Dempster et al., 1977) algorithm, particularly in the learning of Gaussian Mixture Models (GMM) (Redner and Walker, 1984). EM is an iterative method for finding parameter estimates in statistical models than contain unobserved latent variables, alternating between expectation (E) and maximization (M) steps. The expectation (E) step computes the expected value of the log-likelihood using the current estimate for the latent variables. The maximization (M) step computes the parameters that maximize the expected log-likelihood found on the $E$ step. These parameter values determine the latent variable distribution in the next $E$ step. In Gaussian Mixture Models, the EM algorithm is applied to find a set of Gaussian distributions that best fits a set of data points. The $E$ step computes the expected membership of each data point to one of the Gaussian distributions, while the $M$ step computes the parameters for each distribution given the memberships computed in the $E$ step. Then, the $E$ step is repeated with the updated parameters to recompute new membership values. The entire procedure is repeated until model parameters converge.

Despite the mathematical differences, the concept behind ICE is essentially the same. The problem of object recognition in the presence of severe clutter and/or repeated objects can be interpreted as one of estimation of model parameters - the pose of a set of objects-, where the model depends on unobserved latent variables - the correspondences of image features to particular object instances-. Under this perspective, the Clustering step of ICE computes the expected membership of each local feature to one of the object instances, while the Estimation step computes the best object poses given the feature memberships computed in the Clustering step. Then, the entire procedure is repeated until convergence. If our object models were Gaussian distributions, ICE and GMMs would be virtually equivalent.

\section{The MOPED Framework}

This section contains a brief summary of the MOPED framework and its components. Each individual component is explored in depth in subsequent sections.

The steps itemized below compose the basic MOPED framework for the typical requirements of a robotics application. In essence, MOPED is comprised of a single feature extraction and matching step per image, and multiple iterations of Iterative Clustering-Estimation (ICE) that efficiently perform object recognition and pose estimation per object in a bottom-up approach. Assuming the most common setup of object recognition, utilizing a single or a small set of images (i.e. less than 10), we fix ICE to compute two full Clustering-Estimation iterations plus a final cluster merging to remove potential multiple detections that might have not yet converged. This way, we ensure a good trade-off between high recognition rate and reduced system latency, but a greater number of iterations should be considered if working with a larger set of simultaneous images.

1. Feature Extraction. Salient features are extracted from each image. We represent images $\mathbf{I}$ as sets of local features $\mathbf{f}_{m}$. Each image $I_{m} \in \mathbf{I}$ is processed independently, so that

$$
I_{m}=\left\{K_{m}, T_{m}, \mathbf{f}_{m}\right\} \quad \mathbf{f}_{m}=\text { FeatExtract }\left(\mathbf{g}_{m}\right) .
$$

Each individual local feature $f_{j ; m}$ from image $m$ is defined by a $2 \mathrm{D}$ point location $p_{j ; m}=[x, y]^{T}$ and its corresponding feature descriptor $d_{j ; m}$; that is,

$$
\mathbf{f}_{m}=\left\{f_{1 ; m}, \ldots, f_{j ; m}, \ldots, f_{J ; m}\right\} \quad f_{j ; m}=\left\{p_{j ; m}, d_{j ; m}\right\} .
$$

We define the union of all extracted local features from all images $m$ as $\mathbf{f}=\bigcup_{m=1}^{M} \mathbf{f}_{m}$.

2. Feature Matching. One-to-one correspondences are created between extracted features in the image set and object features stored in the database. For efficiency, approximate matching techniques can be used, at the cost of a decreased recognition rate. Let $C$ be a correspondence between an image feature $f_{j ; m}$ and a model feature $F_{i ; o}$, such that

$$
C_{j, m}^{o}= \begin{cases}\left(f_{j ; m}, F_{i ; o}\right), & \text { if } f_{j ; m} \leftrightarrow F_{i ; o} . \\ \emptyset, & \text { otherwise }\end{cases}
$$

The set of correspondences for a given object $o$ and image $m$ is represented as $\mathbf{C}_{m}^{o}=\bigcup_{\forall j} C_{j ; m}^{o}$. The sets of correspondences $\mathbf{C}_{m}, \mathbf{C}^{o}$ are defined equivalently as $\mathbf{C}_{m}=\bigcup_{\forall j, o} C_{j ; m}^{o}$ and $\mathbf{C}^{o}=$ $\bigcup_{\forall j, m} C_{j ; m}^{o}$.

3. Feature Clustering. Features matched to a particular object are clustered in image space $(x, y)$, independently for each image. Given that spatially close features are more likely to belong to the same object instance, we cluster the set of feature locations $\mathbf{p} \in \mathbf{C}_{m}^{o}$, producing a set of clusters that group features spatially close together.

Each cluster $\mathcal{K}_{k}$ is defined by an object identity $o$, an image index $m$, and a subset of the correspondences to object $O$ in image $I_{m}$, that is,

$$
\mathcal{K}_{k}=\left\{o, m, \mathbf{C}_{k} \subset \mathbf{C}_{m}^{o}\right\} .
$$

The set of all clusters is expressed as $\mathcal{K}$.

4. Estimation \#1: Hypothesis Generation. Each cluster is processed in each image independently in search of objects. RANSAC and Levenberg-Marquardt (LM) are used to find object instances that are loosely consistent with each object's geometry in spite of outliers. The number of RANSAC iterations is high and the number of LM iterations is kept low, so that we discover multiple object hypotheses with coarse pose estimation. At this step, each hypothesis $h$ consists of

$$
h=\left\{o, k, T_{h}, \mathbf{C}_{h} \subset \mathbf{C}_{k}\right\},
$$

where $o$ is the object identity of hypothesis $h, k$ is a cluster index, $T_{h}$ is a $4 \times 4$ transformation matrix that defines the object hypothesis pose with respect to the world reference frame, and $\mathbf{C}_{h}$ is the subset of correspondences that are consistent with hypothesis $h$. 
5. Cluster Clustering. As the same object might be present in multiple clusters and images, poses are projected from the image set onto a common coordinate frame, and features consistent with a pose are re-clustered. New, larger clusters are created, that often contain all consistent features for a whole object across the entire image set. These new clusters contain

$$
\mathcal{K}_{K}=\left\{o, \mathbf{C}_{K} \subset \mathbf{C}^{o}\right\} .
$$

6. Estimation \#2: Pose Refinement. After Steps 4 and 5, most outliers have been removed, and each of the new clusters is very likely to contain features corresponding to only one instance of an object, spanned across multiple images. The RANSAC procedure is repeated for a low number of iterations, and poses are estimated using LM with a larger number of iterations to obtain the final poses from each cluster that are consistent with the multi-view geometry.

Each multi-view hypothesis $H$ is defined by

$$
H=\left\{o, T_{H}, \mathbf{C}_{H} \subset \mathbf{C}_{K}\right\},
$$

where $o$ is the object identity of hypothesis $H, T_{H}$ is a $4 \times 4$ transformation matrix that defines the object hypothesis pose with respect to the world reference frame, and $\mathbf{C}_{H}$ is the subset of correspondences that are consistent with hypothesis $H$.

7. Pose Recombination. A final merging step removes any multiple detections that might have survived, by merging together object instances that have similar poses. A set of hypotheses $\mathbf{H}$, with $H_{h}=\left\{o, T_{H}\right\}$, is the final output of MOPED.

\section{$5 \quad$ Addressing Complexity}

In this section, we provide an in-depth explanation of our contributions to address complexity that have been integrated in the MOPED object recognition framework, and how each of our contributions relate to the Iterative Clustering-Estimation procedure.

\subsection{Image Space Clustering}

The goal of Image Space Clustering in the context of object recognition is the creation of object priors based solely on image features. In a generic unstructured scene, it is infeasible to attempt the recognition of objects with no higher-level reasoning than the image-model correspondences $C_{j, m}^{o}=\left(f_{j ; m}, F_{i ; o}\right)$. Correspondences for a single object type $o$ may belong to different object instances, or may be matching outliers. Multicamera setups are even more uncertain, since the amount of image features increases dramatically, and so does the probability of finding multiple repeated objects in the combined set of images. Under these circumstances, the ability to compute a prior over the image features is of utmost importance, in order to evaluate which of the features are likely to belong to the same object instance, and which of them are likely to be outliers.

RANSAC (Fischler and Bolles, 1981) and M-estimators are often the methods of choice to find models in the presence of

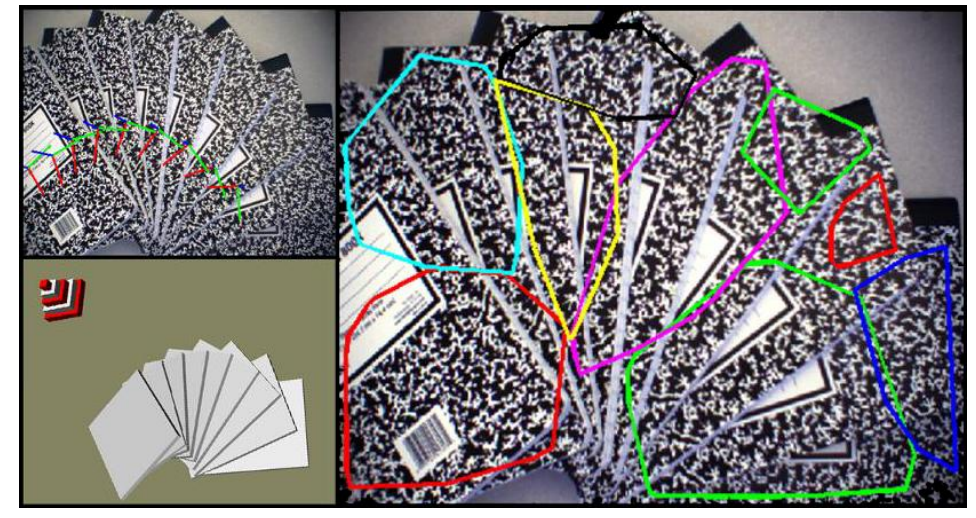

Figure 4: Example of highly cluttered scene and the importance of clustering. (Top-left) Scene with 9 overlapping notebooks. (Bottom-left) Recovered poses for notebooks with MOPED. (Right) Clusters of features in image space.

outliers. However, both of them fail in the presence of heavy clutter and multiple objects, in which only a small percentage of the matched correspondences belong to the same object instance. To overcome this limitation, we propose the creation of object priors based on the density of correspondences across the image, by exploiting the assumption that areas with a higher concentration of correspondences for a given model are more likely to contain an object than areas with very few features. Therefore, we aim to create subsets of correspondences within each image that are reasonably close together and assume they are likely to belong to the same object instance, avoiding the waste of computation time in trying to relate features spread all across the image. We can accomplish this goal by seeking the modes of the density distribution of features in image space. A well-known technique for this task is Mean Shift clustering (Cheng, 1995), which is a particularly good choice for MOPED because no fixed number of clusters needs to be specified. Instead, a radius parameter needs to be chosen, that intuitively selects how close two features must in order to be part of the same cluster. Thus, for each object in each image independently, we cluster the set of feature locations $\mathbf{p} \in \mathbf{C}_{m}^{o}$ (i.e. pixel positions $p=(x, y)$ ), producing a set of clusters $\mathcal{K}$ that contain groups of features spatially close together. Clusters that contain very few features, those in which no object can be recognized, are discarded, thus considering the features as outliers and discarding them as well.

The advantage of using Mean Shift clusters as object priors is illustrated in Fig. 4. In Fig. 4.(top-left) we see an image with 9 notebooks. As a simple example, let us imagine that all notebooks have the same number of correspondences, and that $70 \%$ of those correspondences are correct, i.e., that the global inlier ratio $w=\frac{\# \text { inliers }}{\# \text { points }}=0.7$. The inlier ratio for a particular notebook is then $w_{\mathrm{obj}}=\frac{w}{\# \text { obj }}=0.0778$. The number of iterations $k$ theoretically required (Fischler and Bolles, 1981) to find one particular instance of a notebook with probability $p$ is

$$
k=\frac{\log (1-p)}{\log \left(1-\left(w_{\mathrm{obj}}\right)^{n}\right)},
$$


where $n$ is the number of inliers for a successful detection. If we require $n=5$ inliers and a probability $p=0.95$ of finding a particular notebook, then we should perform $k=1.05 \mathrm{M}$ iterations of RANSAC. On the other hand, clustering the correspondences in smaller sets as in Fig. 4.(right) means that fewer notebooks (at most 3) are present in a given cluster. In such a scenario, finding one particular instance of a notebook with $95 \%$ probability requires 16, 586 and 4386 iterations when 1, 2 and 3 notebooks, resp., are present in a cluster, at least three orders of magnitude lower than the previous case.

\subsection{Estimation \#1: Hypothesis generation}

In the first Estimation step of ICE, our goal is to generate coarse object hypotheses from clusters of features, so that the object poses can be used to refine the cluster associations. In general, each cluster $\mathcal{K}_{k}=\left\{o, m, \mathbf{C}_{k} \subset \mathbf{C}_{m}^{o}\right\}$ may contain features from multiple object hypotheses as well as matching outliers. In order to handle the inevitable presence of matching outliers, we use the robust estimation procedure RANSAC. For a given cluster $\mathcal{K}_{k}$, we choose a subset of correspondences $\mathbf{C} \subset \mathbf{C}_{k}$ and estimate an object hypothesis with the best pose that minimizes the sum of reprojection errors (see Eq. (18)). We minimize the sum of reprojection errors via a standard LevenbergMarquardt (LM) non-linear least squares minimization. If the amount of correspondences in $\mathbf{C}_{k}$ consistent with the hypothesis is higher than a threshold $\epsilon$, we create a new object instance and refine the estimated pose using all consistent correspondences in the optimization. We then repeat this procedure until the amount of unallocated points is lower than a threshold, or the maximum number of iterations has been exceeded. By repeating this procedure for all clusters in all images and objects, we produce a set of hypotheses $\mathbf{h}$, where each hypothesis $h=\left\{o, k, T_{h}, \mathbf{C}_{h} \subset \mathbf{C}_{k}\right\}$.

At this stage, we wish to obtain a set of coarse pose hypotheses to work with, as fast as possible. We require a large number of RANSAC iterations to detect as many object hypotheses as possible, but we can use a low maximum number of LM iterations and loose threshold $\epsilon$ when minimizing the sum of reprojection errors. Accurate pose will be achieved in later stages of MOPED. The initialization of LM for pose estimation can be implemented with either fast $P n P$ solvers such as the ones proposed in (Collet et al., 2009, Lepetit et al., 2008) or even completely at random within the space of possible poses (e.g. some distance in front of the camera). Random initialization is the default choice for MOPED, as it is more robust to the pose ambiguities that sometimes confuse $P n P$ solvers.

\subsection{Hypothesis Quality Score}

It is useful at this point to introduce a robust metric to quantitatively compare the goodness of multiple object hypotheses. The desired object hypothesis metric should favor hypotheses with:

- The most amount of consistent correspondences.
- The minimum distance error in each of the correspondences.

The sum of reprojection errors in Eq. (19) is not a good evaluation metric according to these requirements, as this error is bound to increase whenever an extra correspondence is added. Therefore, the sum of reprojection errors favors hypotheses with the least amount of correspondences, which can lead to choosing spurious hypotheses over more desirable ones.

In contrast, we define a robust estimator based on the Cauchy distribution that balances the two criteria stated above. Consider the set of consistent correspondences $\mathbf{C}_{h}$ for a given object hypothesis, where each correspondence $C_{j}=\left(f_{j ; m}, F_{i ; o}\right)$. Assume the corresponding features in $C_{j}$ have locations in an image $p_{j}$ and in an object model $P_{j}$. Let $d_{j}=d\left(p_{j}, T_{h} P_{j}\right)$ be an error metric that measures the distance between a $2 \mathrm{D}$ point in an image and a 3D point from hypothesis $h$ with pose $T_{h}$ (e.g. reprojection, backprojection errors). Then, the Cauchy distribution $\psi\left(d_{j}\right)$ is defined by

$$
\psi\left(d_{j}\right)=\frac{1}{1+\left(\frac{d_{j}}{\sigma}\right)^{2}},
$$

where $\sigma^{2}$ parameterizes the cut-off distance at which $\psi\left(d_{j}\right)=$ 0.5 . In our case, the distance metric $d_{j}$ is the reprojection error measured in pixels. This distribution is maximal when $d_{j}=0$, i.e. $\psi(0)=1$, and monotonically decreases to zero when a pair of correspondences are infinitely away from each other, i.e. $\psi(\infty)=0$. The Quality Score $Q$ for a given object hypothesis $h$ is then defined as a summation over the Cauchy scores $\psi\left(d_{j}\right)$ for all correspondences:

$$
Q(h)=\sum_{\forall j: C_{j} \in \mathbf{C}_{h}} \psi\left(d_{j}\right)=\sum_{\forall C_{j} \in \mathbf{C}_{h}} \frac{1}{1+\frac{d^{2}\left(p_{j}, T_{h} P_{j}\right)}{\sigma^{2}}} .
$$

The Q-Score has a lower bound at 0 , if a given hypothesis has no correspondences or if all its correspondences have infinite error, and has an upper bound at $|O|$, which is the total number of correspondences for model $O$. This score allows us to reliably rank our object hypotheses and evaluate their strength.

The cut-off distance $\sigma$ may be either a fixed value, or adjusted at each iteration via robust estimation techniques (Zhang, 1997), depending on the application. Robust estimation techniques require a certain minimum outlier/inlier ratio to work properly $\left(\frac{\# \text { outliers }}{\# \text { inliers }}<1\right.$ in all cases $)$, known as the breaking point of a robust estimator (Huber, 1981). In the case of MOPED, the outlier/inlier ratio is often well over the breaking point of any robust estimator, especially when multiple instances of an object are present; as a consequence, robust estimators might result in unrealistically large values of $\sigma$ in complex scenes. Therefore, we choose to set a fixed value for the cut-off parameter, $\sigma=2$ pixels, for a good balance between encouraging a large number of correspondences while keeping their reprojection error low. 


\subsection{Cluster Clustering}

The disadvantage of separating the image search space into a set of clusters is that the produced pose hypotheses may be generated with only partial information from the scene, given that information from other clusters and other views is not considered in the initial Estimation step of ICE. However, once a rough estimate of the object poses is known, we can merge the information from multiple clusters and multiple views to obtain sets of correspondences that contain all features from a single object instance (see Fig. 3).

Multiple alternatives are available to group similar hypotheses into clusters. In this section, we propose a novel hypothesis clustering algorithm called Projection Clustering, in which we perform correspondence-level grouping from a set of object hypotheses $\mathbf{h}$ and provide a mechanism to robustly filter any pose outliers.

For comparison, we introduce a simpler Cluster Clustering scheme based on Mean Shift, and analyze the computational complexity of both schemes to conclude in which cases we might prefer one over the other.

\subsubsection{Mean Shift clustering on pose space}

A straightforward hypothesis clustering scheme is to perform Mean Shift clustering on all hypotheses $h=\left\{o, k, T_{h}, \mathbf{C}_{h} \subset \mathbf{C}_{k}\right\}$ for a given object type $o$. In particular, we cluster the pose hypotheses $T_{h}$ in pose space. In order to properly measure distances between poses, it is convenient to parameterize rotations in terms of quaternions and project them in the same half of the quaternion hypersphere prior to clustering, using then Mean Shift on the resulting 7-dimensional poses. After this procedure, we merge the correspondence clusters $\mathbf{C}_{h}$ of those poses that belong to the same pose cluster $\mathcal{T}_{K}$.

$$
\mathbf{C}_{K}=\bigcup_{T_{h} \in \mathcal{T}_{K}} \mathbf{C}_{h}
$$

This produces clusters $\mathcal{K}_{K}=\left\{o, \mathbf{C}_{K} \subset \mathbf{C}^{o}\right\}$ whose correspondence clusters span over multiple images. In addition, the centroid of each pose cluster $\mathcal{T}_{K}$ can be used as initialization for the following Estimation iteration of ICE. At this point, we can discard all correspondences not consistent with any pose hypothesis, thus filtering many outliers and reducing the search space for future iterations of ICE. The computational complexity of Mean Shift is $\mathcal{O}\left(d N^{2} t\right)$, where $d=7$ is the dimensionality of the clustered data, $N=|\mathbf{h}|$ is the total number of hypotheses to cluster, and $t$ is the number of iterations that Mean Shift requires. In practice, the number of hypotheses is often fairly small, and $t \leq 100$ in our implementation.

\subsubsection{Projection clustering}

Mean Shift provides basic clustering in pose space, and works well when multiple correct detections of an object are present. However, it is possible that spurious false positives are detected in the hypothesis generation step. It is important to realize that these false positives are very rarely exclusively due to random outliers in the feature matching process. To the contrary, most outlier detections are artifacts of the projection of a 3D scene into a 2D image when captured by a perspective camera. In particular, we can distinguish two different cases:

- A group of correct matches whose 3D configuration is degenerate or near-degenerate (e.g. a group of 3D points that are almost collinear), incorrectly grouped with one single matching outlier. In this case, the output pose is largely determined by the location of the matching outlier, which causes arbitrarily erroneous hypotheses to be accepted as correct.

- Pose ambiguities in objects with planar surfaces. The sum of reprojection errors in planar surfaces may contain two complementary local minima in some configurations of pose space(Schweighofer and Pinz, 2006), which often causes the appearance of two distinct and partially overlapping object hypotheses. These hypotheses are usually too distant in pose space to be grouped together.

The false positives output in the pose hypothesis generation are often too distant from any correct hypothesis in the scene, and cannot be merged using regular clustering techniques (e.g. Mean Shift). However, the point features that generated those false positives are usually correct, and they can provide valuable information to some of the correct object hypotheses. In Projection clustering, we process each point feature individually, and assign them to the strongest pose hypothesis to which they might belong. Usually, spurious poses only contain a limited number of consistent point features, thus resulting in lower Qscores (Section 5.3) than correct object hypotheses. By transferring most of the point features to the strongest object hypotheses, we not only utilize the extra information available in the scene for increased accuracy, but also filter most false positives by lowering their number of consistent points below the required minimum.

The first step in Projection clustering is the computation of Q-Scores for all object hypotheses $\mathbf{h}$. We generate a pool of potential correspondences $\mathbf{C}_{o}$ that contains all correspondences for a given object type that are consistent with at least one pose hypothesis. Correspondences from all images are included in $\mathbf{C}_{o}$. We compute the Quality score for each hypothesis $h$ from all correspondences $C_{j}=\left(f_{j}, F_{j}\right)$ such that $C_{j} \in \mathbf{C}_{o}$.

$$
Q(h)=\sum_{\forall j: C_{j} \in \mathbf{C}_{o}} \psi\left(d_{j}\right)=\sum_{\forall C_{j} \in \mathbf{C}_{o}} \frac{1}{1+\frac{d^{2}\left(p_{j}, T_{h} P_{j}\right)}{\sigma^{2}}}
$$

For each potential correspondence $C_{j}$ in $\mathbf{C}_{o}$, we define a set of likely hypotheses $\mathbf{h}_{j}$ as the set of those hypotheses $h$ whose reprojection error is lower than a threshold $\gamma$. This threshold can be interpreted as an attraction coefficient; large values of $\gamma$ lead to heavy transference of correspondence to strong hypotheses, while small values cause few correspondences to transfer from one hypothesis to another. In our experiments, a large threshold $\gamma$ of 64 pixels is used. 


$$
h \in \mathbf{h}_{j} \Longleftrightarrow d^{2}\left(p_{j}, T_{h} P_{j}\right)<\gamma
$$

At this point, the relation between correspondences $C_{j}$ and hypotheses $h$ is broken. In other words, we empty the set of correspondences $\mathbf{C}_{h}$ for each hypothesis $h$, so that $\mathbf{C}_{h}=\emptyset$. Then, we re-assign each correspondence $C_{j} \in \mathbf{C}_{o}$ to the pose hypothesis $h$ within $\mathbf{h}_{j}$ with stronger overall Q-Score:

$$
\mathbf{C}_{h} \leftarrow C_{j}: h=\underset{h \in \mathbf{h}_{j}}{\arg \max } Q(h)
$$

Finally, it is important to check the remaining number of correspondences that each object hypothesis has after Projection clustering. Pose hypotheses that retain less than a minimum number of consistent correspondences are considered outliers and therefore discarded.

The Projection Clustering algorithm we propose has a computational complexity $\mathcal{O}(M N I)$, where $M=|\mathbf{C}|$ is the total number of correspondences to process, $N=|\mathbf{h}|$ is the number of pose hypotheses and $I=|\mathbf{I}|$ is the total number of images. Comparing this with the complexity $\mathcal{O}\left(d N^{2} t\right)$ of Mean Shift, we see that the only advantage of Mean Shift in terms of computational cost would be in the case of object models with enormous numbers of features and many high resolution images, so that $\mathcal{O}(M N I) \gg \mathcal{O}\left(d N^{2} t\right)$. While offering a similar computational complexity, the advantage of Projection Clustering with respect to Mean Shift is in terms of improved robustness and outlier detection, both of which are essential for handling increased complexity in MOPED.

\subsubsection{Performance Comparison}

In this experiment, we compare the recognition performance of MOPED when using the two Cluster Clustering approaches explained in this section. In addition, and given that Projection Clustering depends on the behavior of a hypothesis ranking mechanism, we implement four different ranking mechanisms and compare their performance. The first ranking mechanism is our own Q-Score introduced in Section 5.3, while the other approaches considered are the sum of reprojection errors, the average reprojection error, and the number of consistent correspondences. The performance of MOPED when using the different Cluster Clustering and hypothesis ranking algorithms is shown in Table 1, on a subset of 100 images from the Simple Movie Benchmark (Section 6.2.3) that contain a total of 1289 object instances.

An object hypothesis is considered a true positive if its pose estimate is within $5 \mathrm{~cm}$ and 10 degrees of the ground truth pose. Object hypotheses whose pose is outside this error range are considered false positives. Ground truth objects without an associated true positive are considered false negatives. According to these performance metrics, the appearance of pose ambiguities and misdetections is particularly critical, since they often produce both a false positive - the rotational error is greater than the threshold- and a false negative - no pose agrees with the ground truth-.

The overall best-performer is Projection Clustering when using Q-Score as its hypothesis ranking metric, which correctly
Table 1: Mean Recognition per Image: Mean Shift vs Projection Clustering, in the Simple Movie Benchmark (see Section 6.2 .3 for details).

\begin{tabular}{rccc}
\hline & True Pos. & False Pos. & False Neg. \\
\hline Mean Shift & 10.92 & 3.32 & 1.97 \\
P.Clust. (Q-Score) & $\mathbf{1 1 . 3}$ & $\mathbf{2 . 2 9}$ & $\mathbf{1 . 5 9}$ \\
P.Clust. (Reproj) & 11.05 & 7.4 & 1.84 \\
P.Clust. (Avg Reproj) & 10.88 & 7.81 & 2.01 \\
P.Clust. (\# Corresp) & 3.6 & 9.82 & 9.29 \\
\hline
\end{tabular}

recognizes and estimates the pose of $87.6 \%$ of the objects in the dataset. Mean Shift is the second best performer, but the increased false positive rate is mainly due to pose ambiguities that cannot be resolved and produce spurious detections. The different hypothesis ranking schemes critically impact the overall performance of Projection Clustering, as multiple poses often need to be merged after the first Clustering-Estimation iteration. Ranking spurious poses over correct poses results in an increased number of false positives, as Projection Clustering is unable to merge object hypothesis properly. While Q-Score is able to estimate the best pose out a set of multiple detections, Reprojection and Avg. Reprojection select sub-optimal poses that contain just a few points, often including outliers. This behavior severely impacts the performance of Projection Clustering, which barely merges any pose hypotheses and produces an increased number of false positives. The Num. Correspondences metric prefers hypotheses with many consistent matches in the scene, and Projection Clustering merges hypotheses correctly. Unfortunately, the chosen best hypotheses are in most cases incorrect due to ambiguities, estimating only $28 \%$ of the poses correctly. It must be noted that in simple scenes with a few unoccluded objects all the evaluated metrics perform similarly well. Projection Clustering and Q-Score, however, showcase increased robustness when working with the most complex scenes.

\subsection{Estimation \#2: Pose Refinement}

At the second iteration of ICE, we use the information from initial object hypotheses to obtain clusters that are most likely to belong to a single object instance, with very few outliers. For this reason, the Estimation step at the second iteration of ICE requires only a low number of RANSAC iterations to find object hypotheses. In addition, we use a high number of LM iterations to estimate object poses accurately.

This procedure is equivalent to that of the first Estimation step, being the objective function to minimize the only difference between the two. For a given multi-view feature cluster $\mathcal{K}_{K}$, we perform RANSAC on subsets of correspondences $\mathbf{C} \subset \mathbf{C}_{K}$ and obtain pose hypotheses $H=\left\{o, T_{H}, \mathbf{C}_{H} \subset \mathbf{C}_{K}\right\}$ with consistent correspondences $\mathbf{C}_{H} \subset \mathbf{C}_{K}$. The objective function to minimize can be either the sum of reprojection er- 
rors (Eq. (19)) or the sum of backprojection errors (Eq. (24)). See Appendix A for a discussion on these two objective functions.

We initialize the non-linear minimization of the chosen objective function using the highest-ranked pose in $\mathbf{C}_{K}$ according to their Q-Scores. This non-linear minimization is performed, again, with a standard Levenberg-Marquardt non-linear least squares minimization algorithm.

\subsection{Truncating ICE: Pose Recombination}

An optional final step should be applied in case ICE has not converged after two iterations, in order to remove multiple detections of objects. In this case, we perform a full Clustering step as in Section 5.4, and if any hypothesis $H \in \mathbf{H}$ is updated with transferred correspondences, we perform a final LM optimization that minimizes Eq. (24) on all correspondences $\mathbf{C}_{H}$.

\section{Addressing Scalability and Latency}

In this section, we present multiple contributions to optimize MOPED in terms of scalability and latency. We first introduce a set of four Benchmarks designed to stress test every component of MOPED. Each of our contributions is evaluated and verified on this set of benchmarks.

\subsection{Baseline system}

For comparison purposes, we provide results for a Baseline system that implements the MOPED framework with none of the optimizations described in Section 6. In each case, we have tried to choose the most widespread publicly available code libraries for each task. In particular, the following configuration is used as the baseline for our performance experiments:

Feature Extraction with an OpenMP-enabled, CPUoptimized version of SIFT we have developed.

Feature matching with a publicly available implementation of ANN by Arya et al. (1998) and 2-NN Per Object (described in Section 6.3.1).

Image Space clustering with a publicly available C implementation of Mean Shift (Dollár and Rabaud, 2010).

Estimation \#1 with 500 iterations of RANSAC and up to 100 iterations of the Levenberg-Marquardt (LM) implementation from Lourakis (2010), optimizing the sum of reprojection errors in Eq. (18).

Cluster Clustering with Mean Shift, as described in Section 5.4.1.

Estimation \#2 with 24 iterations of RANSAC and up to 500 iterations of LM, optimizing the sum of backprojection errors in Eq. (24). The particular number of iterations of RANSAC in this step is not a critical factor, as most objects are successfully recognized in the first $10-15$ iterations. It is useful, however, to use a multiple of the number of concurrent threads (see Section 6.4.3) for performance reasons.

Pose Recombination with Mean Shift and up to 500 iterations of LM.

\subsection{Benchmarks}

We present four benchmarks (Fig. 5) designed to stress test every component of our system. All benchmarks, both synthetic and real-world, provide exclusively a set of images and ground truth object poses (i.e., no synthetically computed feature locations or correspondences). We performed all experiments on a $2.33 \mathrm{GHz}$ quad-core Intel(R) Xeon(R) E5345 CPU, 4 GB of RAM and a nVidia GeForce GTX 260 GPU running Ubuntu 8.04 (32 bits).

\subsubsection{The Rotation Benchmark}

The Rotation Benchmark is a set of synthetic images that contains highly cluttered scenes with up to 400 cards in different sizes and orientations. This benchmark is designed to test MOPED's scalability with respect to the database size, while keeping a constant number of features and objects. We have generated a total of 100 independent images for different resolutions $(1400 \times 1050,1000 \times 750,700 \times 525,500 \times 375$ and $350 \times 262$ ). Each image contains from 5 to 80 different objects and up to 400 simultaneous object instances.

\subsubsection{The Zoom Benchmark}

The Zoom Benchmark is a set of synthetic images that progressively zooms in on 160 cards until only 12 cards are visible. This benchmark is designed to check the scalability of MOPED with respect to the total number of detected objects in a scene. We generated a total of 145 independent images for different resolutions $(1400 \times 1050,1000 \times 750,700 \times 525,500 \times 375$ and $350 \times 262$ ). Each image contains from 12 to 80 different objects and up to 160 simultaneous object instances. This benchmark simulates a board with 160 cards seen by a $60^{\circ} \mathrm{FOV}$ camera at distances ranging from $280 \mathrm{~mm}$ to $1050 \mathrm{~mm}$. The objects were chosen to have the same number of features at each scale. Each image has over 25000 features.

\subsubsection{The Simple Movie Benchmark}

Synthetic benchmarks are useful to test a system in controlled conditions, but are a poor estimator of the performance of a system in the real world. Therefore, we provide two real-world scenarios for algorithm comparison. The Simple Movie Benchmark consists of a 1900-frame movie at 1280 x 720 resolution, each image containing up to 18 simultaneous object instances.

\subsubsection{The Complex Movie Benchmark}

The Complex Movie Benchmark consists of a 3542-frame movie at $1600 \times 1200$ resolution, each image containing up to 60 simultaneous object instances. The database contains 91 models and 47342 SIFT features when running this benchmark. It is noteworthy that the scenes in this video present particularly complex situations, including: several objects of the same model contiguous to each other, which stresses the clustering step; overlapping partially-occluded objects, which stresses RANSAC; and objects in particularly ambiguous poses, which 


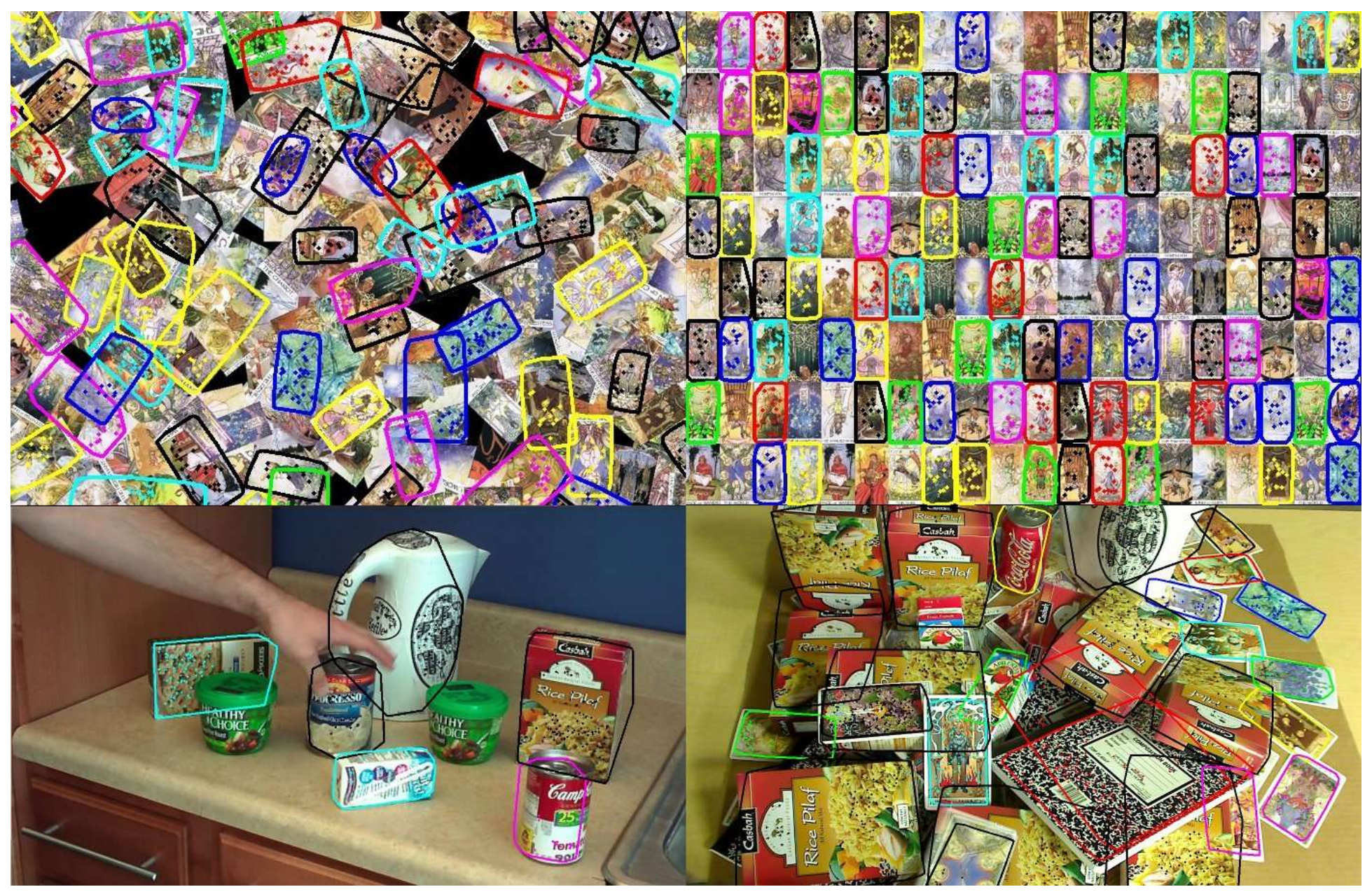

Figure 5: MOPED Benchmarks. For the sake of clarity, only half of the detected objects are marked. (a) The Rotation Benchmark: MOPED processes this scene 36.4x faster than the Baseline. (b) The Zoom Benchmark: MOPED processes this scene 23.4x faster than the Baseline. (c) The Simple Movie Benchmark. (d) The Complex Movie Benchmark.

stresses both LM and the merging algorithm, that encounter difficulties determining which pose is preferable.

\subsection{Feature Matching}

Once a set of features have been extracted from input image(s), we must find correspondences between the image features and our object database. Matching is done as a nearest neighbor search in the 128-dimensional space of SIFT features. An average database of 100 objects can contain over 60,000 features. Each input image, depending on the resolution and complexity of the scene, can contain over 10,000 features.

The feature matching step aims to create one-to-one correspondences between model and image features. The feature matching step is, in general, the most important bottleneck for model-based object recognition to scale to large object databases. In this section, we propose and evaluate different alternatives to maximize scalability with respect to the number of objects in the database, without sacrificing accuracy. The extension of the matching search space is, in this case, the balancing factor between accuracy and speed when finding nearest neighbors.

There are no known exact algorithms for solving the matching problem that are faster than linear search. Approximate algorithms, on the other hand, can provide massive speedups at the cost of a decreased matching accuracy, and are often used wherever speed is an issue. Many approximate approaches for finding the nearest neighbors to a given feature are based on $k d$-trees (e.g. ANN (Arya et al., 1998), randomized kd-trees (Silpa-Anan and Hartley, 2008), FLANN (Muja and Lowe, 2009)) or hashing (e.g. LSH (Andoni and Indyk, 2006)). A ratio test between the first two nearest neighbors is often performed for outlier rejection. We analyze the different alternatives in which these techniques are often applied to feature matching, and propose an intermediate solution that achieves a good balance between recognition rate and system latency.

\subsubsection{2-NN per Object}

On the one end, we can compare the image features against each model independently. If using e.g. ANN, we build a kd-tree for each model in the database once (off-line), and we match each of 
them against every new image. This process entails a complexity of $\mathcal{O}\left(\left|\mathbf{f}_{m}\right||\mathbf{O}| \log \left(\left|\overline{\mathbf{F}}_{o}\right|\right)\right)$, where $\left|\mathbf{f}_{m}\right|$ is the number of features on the image, $|\mathbf{O}|$ the number of models in the database, and $\left|\overline{\mathbf{F}}_{o}\right|$ the mean number of features for each model. When $|\mathbf{O}|$ is large, this approach is vastly inefficient as the cost of accessing each kd-tree dominates the overall search cost. The search space is in this case very limited, and there is no degradation in performance when new models are added to the database. We refer to it as $O B J_{-} M A T C H$.

\subsubsection{2-NN per Database}

On the other end, we can compare the image features against the whole object database. This naïve alternative, which we term $D B_{-} M A T C H$, builds just one $k d$-tree containing the features from all models. This solution has a complexity of $\mathcal{O}\left(\left|\mathbf{f}_{m}\right| \log \left(|\mathbf{O}|\left|\overline{\mathbf{F}}_{o}\right|\right)\right)$. The search space is in this case the whole database. While this approach is orders of magnitude faster than the previous one, every new object added to the database degrades the overall recognition performance of the system due to the presence of similar features in different objects. In the limit, if an infinite number of objects were added to the database, no correspondences would ever be found, because the ratio test between the first and second nearest neighbor would be always close to 1 .

\subsubsection{Brute Force on GPU}

The advent of GPUs and their many-core architecture allows the efficient implementation of an exact feature matching algorithm. The parallel nature of the brute force matching algorithm suits the GPU, and allows it to be faster than the ANN approach when $|\mathbf{O}|$ is not too large. Given that this algorithm scales linearly with the number of features instead of logarithmically, we can match each model independently without performance loss.

\subsection{4 k-NN per Database}

Alternatively, one can consider the closest $k$ nearest neighbors instead (with $k>2$ ). k-ANN implementations using kdtrees can provide more neighbors without significantly increasing their computational cost, as they are often a byproduct of the process of obtaining the nearest neighbor. An intermediate approach to the ones presented before is the search for $k$ multiple neighbors in the whole database. If two neighbors from the same model are found, the distance ratio is then applied to the 2 nearest neighbors from the same model. If the nearest neighbor is the only neighbor for a given model, we apply the distance ratio with the second nearest neighbor to avoid spurious correspondences. This algorithm (with $k=90$ ) is the default choice for MOPED.

\subsubsection{Performance comparison}

Fig. 6 compares the cost of the different alternatives on the Rotation Benchmark. OBJ_MATCH and GPU scale linearly with respect to $|\mathbf{O}|$, while $D B_{-} M A T C H$ and MOPED-k scale
Table 2: Feature matching algorithms in the Simple Movie Benchmark. GPU used as performance baseline, as it computes exact nearest neighbors.

\begin{tabular}{|c|c|c|c|}
\hline \# Corresp: & After Matching & After clustering & Final \\
\hline GPU & 3893.7 & 853.2 & 562.1 \\
\hline OBJ_MATCH & 3893.6 & 712.0 & 449.2 \\
\hline DB_MATCH & 1778.4 & 508.8 & 394.7 \\
\hline MOPED-90 & 3624.9 & 713.6 & 428.9 \\
\hline & Matching Time $(\mathrm{ms})$ & \multicolumn{2}{|c|}{ Objects Found } \\
\hline GPU & 253.34 & \multicolumn{2}{|l|}{8.8} \\
\hline OBJ_MATCH & 498.586 & \multicolumn{2}{|l|}{8.0} \\
\hline DB_MATCH & 129.85 & \multicolumn{2}{|l|}{7.5} \\
\hline MOPED-90 & 140.36 & \multicolumn{2}{|l|}{8.2} \\
\hline
\end{tabular}
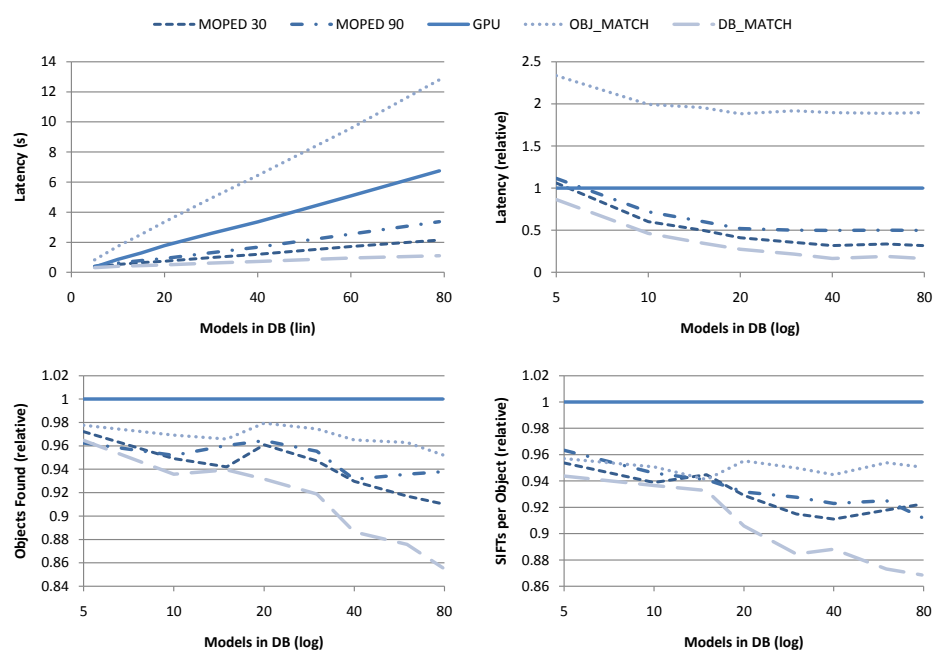

Figure 6: Scalability of feature matching algorithms with respect to the database size, in the Rotation Benchmark at $1400 \times 1050$ resolution.

almost logarithmically. We show MOPED-k using $k=90$ and $k=30$. The value of $k$ adjusts the speed and quality behavior of MOPED between $O B J_{-} M A T C H(k=\infty)$ and $D B_{-} M A T C H$ $(k=2)$. The recognition performance of MOPED-k when using the different strategies is shown in Table 2. GPU provides an upper bound for the object recognition, as it is an exact search method. OBJ_MATCH comes closest in raw matching accuracy with MOPED-90 a close second. However, the number of objects detected are nearly the same. The matching speed of MOPED-90 is, however, significantly better than $O B J_{-} M A T C H$. Feature matching in MOPED-90 thus provides a significant performance increase without sacrificing much accuracy. 


\subsection{Architecture Optimizations}

Our algorithmic improvements were focused mainly on boosting the scalability and robustness of the system. The architectural improvements of MOPED are obtained as a result of an implementation designed to make the best use of all the processing resources of standard compute hardware. In particular, we use GPU-based processing, intra-core parallelization using SIMD instructions, and multi-core parallelization. We have also carefully optimized the memory subsystem, including bandwidth transfer and cache management.

All optimizations have been devised to reduce the latency between the acquisition of an image and the output of the pose estimates, to enable faster response times from our robotic platform.

\subsubsection{GPU and Embarrassingly Parallel Problems}

State-of-the-art CPUs, such as the Intel Core i7 975 Extreme, can achieve a peak performance of 55.36 GFLOPS, according to the manufacturer (Intel Corp., 2010). State-of-the-art GPUs, such as the ATI Radeon HD 5900, can achieve a peak performance of 4640 SP GFLOPS (AMD, 2010).

To use GPU resources efficiently, input data needs to be transferred to the GPU memory. Then, algorithms are executed simultaneously on all shaders, and finally recover the results from the GPU memory. As communication between shaders is expensive, the best GPU-performing algorithms are those that can be divided evenly into a large number of simple tasks. This class of easily separable problems is called Embarrassingly Parallel Problems (EPP) (Wilkinson and Allen, 2004).

GPU-Based Feature Extraction. Most feature extraction algorithms consist of an initial keypoint detection step followed by a descriptor calculation for each keypoint, both of which are EPP. Keypoint detection algorithms can process each pixel from the image independently. They may need information about neighboring pixels, but they do not typically need results from them. After obtaining the list of keypoints, the respective descriptors can also be calculated independently.

In MOPED, we consider two of the most popular locally invariant features: SIFT (Lowe, 2004) and SURF (Bay et al., 2008). SIFT features have proven to be among the bestperforming invariant descriptors in the literature (Mikolajczyk and Schmid, 2005), while SURF features are considered to be a fast alternative to SIFT. MOPED uses SIFT-GPU (Wu, 2007) as its main feature extraction algorithm. If compatible graphics hardware is not detected MOPED automatically reverts back to performing SIFT extraction on the CPU, which is an OpenMPenabled, CPU-optimized version of SIFT we have developed. A GPU-enabled version of SURF, GPU-SURF (Cornelis and Van Gool, 2008), is used for comparison purposes.

We evaluate the latency of the three implementations in Fig. 7. The comparison is as expected: GPU versions of both SIFT and SURF provide tremendous improvements over their non-GPU counterparts. Table 3 compares the object recognition performance of SIFT and SURF: SURF proves to be $2.59 \mathrm{x}$
Table 3: SIFT vs. SURF: Mean Recognition Performance per Image in Zoom Benchmark.

\begin{tabular}{ccc}
\hline & Latency $(\mathrm{ms})$ & Recognized Objects \\
\hline SIFT & 223.072 & 13.83 \\
SURF & 86.136 & 6.27 \\
\hline
\end{tabular}

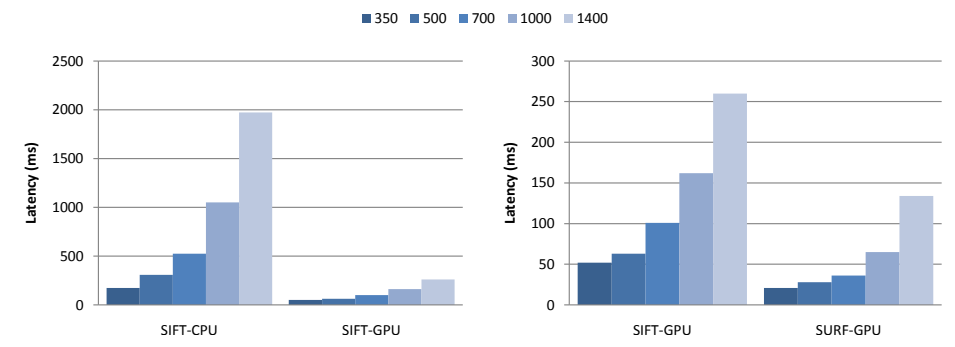

Figure 7: SIFT-CPU vs. SIFT-GPU vs. SURF-GPU, in the Rotation Benchmark at different resolutions. (left) SIFT-CPU vs. SIFT-GPU: $658 \%$ speed increase in SIFT extraction on GPU. (right) SIFT-GPU vs. SURF-GPU: $91 \%$ speed increase in SURF over SIFT at the cost of lower matching performance.

faster than SIFT at the cost of detecting $54 \%$ less objects. In addition, the performance gap between both methods decreases significantly as image resolution increases, as shown in Fig. 7. For MOPED, we consider SIFT to be almost always the better alternative when balancing recognition performance and system latency.

GPU Matching. Performing feature matching in the GPU requires a different approach than the standard Approximate Nearest Neighbor techniques. Using ANN, each match involves searching in a kd-tree, which requires fast local storage and a heavy use of branching that are not suitable for GPUs.

Instead of using ANN, Wu (2007) suggests the use of brute force nearest neighbor search on the GPU, which scales quite well as vector processing matches the GPU structure quite well. In Fig. 6, brute force GPU matching is shown to be faster than per-object ANN and provide better quality matches because it is not approximate. As graphics hardware becomes cheaper and more powerful, brute-force feature matching in large databases might become the most sensible choice in the near future.

\subsubsection{Intra-core optimizations}

SSE instructions allow MOPED to perform 12 floating point instructions per cycle instead of just one. The $3 \mathrm{D}$ to $2 \mathrm{D}$ projection function, critical in the pose estimation steps, is massively improved by using SSE-specific algorithms from Van Weveren (2005) and Conte et al. (2000).

The memory footprint of MOPED is very lightweight for current computers. In the case of a database of 100 models and a total of 102.400 SIFT features, the required memory is less than 13MB. Runtime memory footprint is also small: a scene 


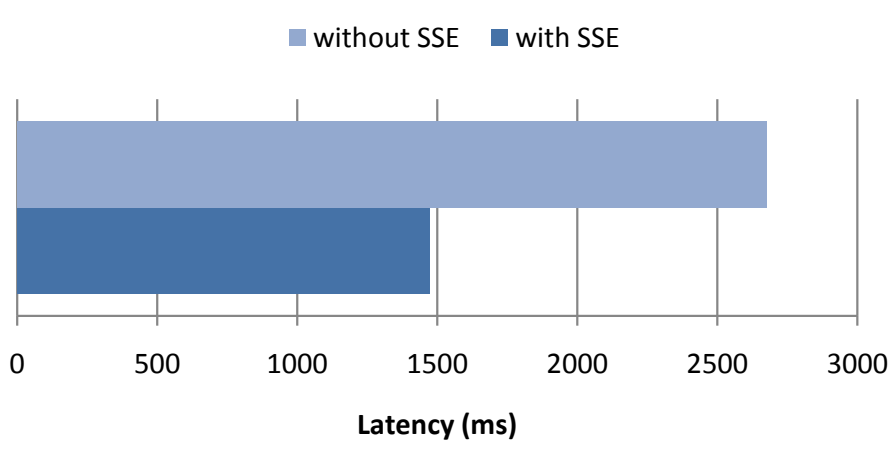

Figure 8: SSE performance improvement in the Complex Movie Benchmark. Time per frame without counting SIFT extraction.

with 100 different objects with 100 matched features each would require less than $10 \mathrm{MB}$ of memory to be processed. This is possible thanks to using dynamic and compact structures, such as lists and sets, and removing unused data as soon as possible. In addition, SIFT descriptors are stored as integer numbers in a 128-byte array instead of a 512-byte array. Cache performance has been greatly improved due to the heavy use of memoryaligned and compact data structures (Dysart et al., 2004).

The main data structures are kept constant throughout the algorithm, so that no data needs to be copied or translated between steps. k-ANN feature matching benefits from compact structures in the kd-tree storage, as smaller structures increase the probability of staying in the cache for faster processing. In image space clustering, the performance of Mean Shift is boosted 250 times through the use of compact data structures.

The overall performance increase is over $67 \%$ in CPU processing tasks (see Fig. 8).

\subsubsection{Symmetric Multiprocessing}

Symmetric Multiprocessing (SMP) is a multiprocessor computer architecture with identical processors and shared memory space. Most multi-core based computers are SMP systems. OpenMP is a standard framework for multi-processing in SMP systems that we implement in MOPED.

We use Best Fit Decreasing (Johnson, 1974) to balance the load between cores using the size of a cluster as an estimate of its processing time, given that each cluster of features can be processed independently. Tests on a subset of 10 images from the Complex Movie Benchmark show performance improvements of $55 \%$ and $174 \%$ on dual and quad core CPUs respectively, as seen in Fig. 9.

\subsubsection{Multi-Frame Scheduling}

In order to maximize the system throughput, MOPED can benefit from GPU-CPU pipeline scheduling (Chatha and Vemuri, 2002). In order to use all available computing resources, a second execution thread can be added, as shown in Fig. 10. However, the GPU and CPU execution times are not equal in real scenes, and one of the execution threads often needs to wait for

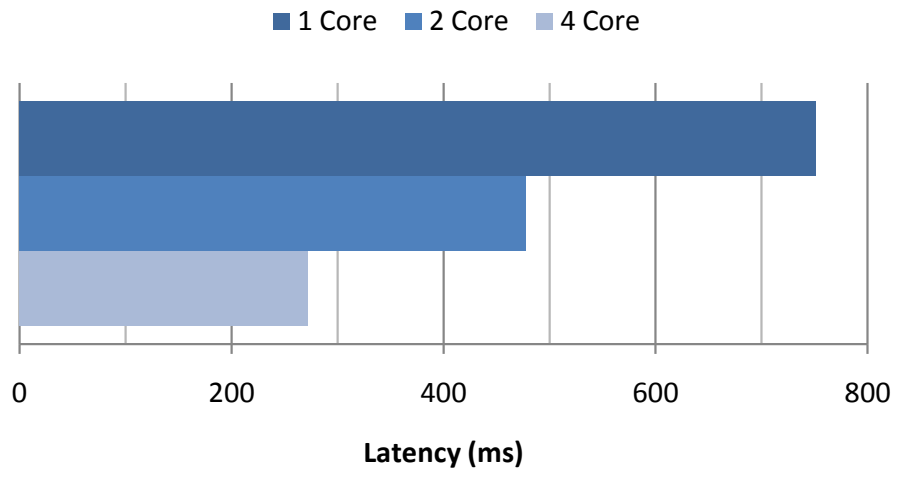

Figure 9: Performance improvement of Pose Estimation step in multi-core CPUs, in the Complex Movie Benchmark.

Table 4: Impact of pipeline scheduling, in the Simple Movie Benchmark. Latency measurements in ms.

\begin{tabular}{rccc}
\hline & FPS & Latency & Latency Sd \\
\hline Sched. MOPED & $\mathbf{3 . 4 9}$ & 368.45 & 92.34 \\
Non-Sched. MOPED & 2.70 & $\mathbf{3 0 3 . 2 3}$ & $\mathbf{6 9 . 2 6}$ \\
Baseline & 0.47 & 2124.30 & 286.54 \\
\hline
\end{tabular}

the other (see Fig. 11). The impact of pipeline scheduling depends heavily on image resolution, as shown in Fig. 12, because the GPU and CPU loads do not increase at the same rate when increasing the number of features but keeping the same number of objects in each scene. In MOPED, pipeline scheduling may increase latency significantly, especially if using high resolution images, but also increases throughput almost two-fold. Since GPU processing is the bottleneck on very small resolutions, these are the best scenarios for pipeline scheduling. For example, as seen in Fig. 12, at a lower resolution of $500 \times 380$, throughput is increased by $95.6 \%$ and latency is increased by $9 \%$.

We further test the impact of pipeline scheduling in a real sequence in the Simple Movie Benchmark, in Table 4. The average throughput of the overall system is increased by $25 \%$ when using pipeline scheduling, at the cost of $21.5 \%$ more latency. In addition, we see the average system latency fluctuates $33.2 \%$ more when using pipeline scheduling. In our particular application, latency is a more critical factor than throughput, as our robot HERB (Srinivasa et al., 2010) must interact with the world in real time. Therefore, we choose not to use pipeline scheduling in our default implementation of MOPED (and in the experiments displayed in this paper). In general, pipeline scheduling should be implemented in any kind of offline process, or whenever latency is not a critical factor in object recognition.

\subsection{Performance evaluation}

In this section, we evaluate the impact of our combined optimizations on the overall performance of MOPED, compared 


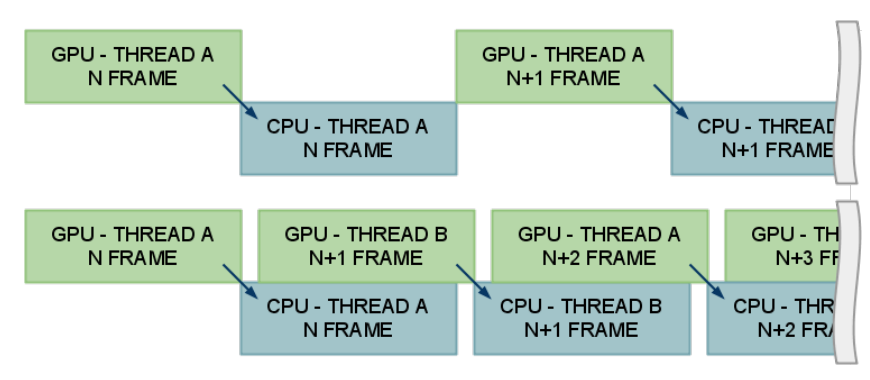

Figure 10: (top) Standard MOPED uses the GPU to obtain the SIFT features, then the CPU to process them. (bottom) Addition of a second execution thread does not substantially increase the system latency.

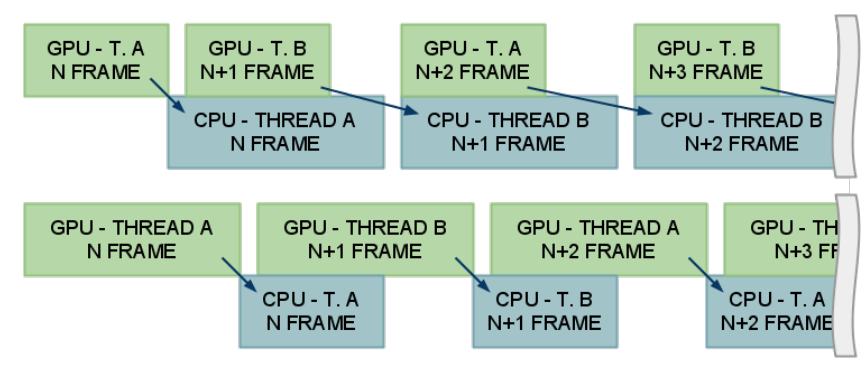

Figure 11: (top) Limiting factor: CPU. GPU thread processing frame $\mathrm{N}+1$ must wait for $\mathrm{CPU}$ processing frame $\mathrm{N}$ to finish, increasing latency. (bottom) Limiting factor: GPU. No substantial increase in latency.

to the Baseline system in Section 6.1, and we analyze how the application of our optimizations improves system latency and scalability.

Testing both systems on the Simple Movie Benchmark (Table 4), MOPED outperforms the baseline with a $5.74 \mathrm{x}$ increase in throughput and a $7.01 \mathrm{x}$ decrease in latency. These improvements become more acute the greater the scene complexity is, Our architectural optimizations offer improvement even with the simple scenes, but the overhead of managing the SMP and GPU processing is large enough to limit the improvement. However, in scenes with high complexity (and, therefore, with high number of features and objects) this overhead is negligible, resulting in massive performance boosts. In the Com-
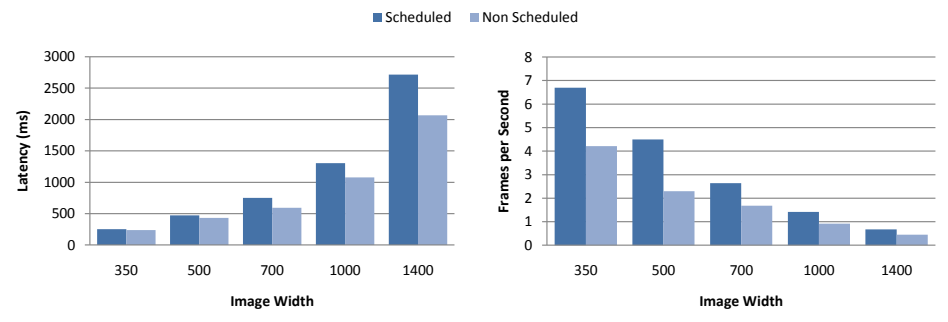

Figure 12: Impact of image resolution in Pipeline Scheduling, in the Rotation Benchmark. (left) Latency comparison (ms). (right) Throughput comparison (FPS). plex Movie Benchmark, MOPED shows an average throughput of 0.44 frames per second and latency of $2173.83 \mathrm{~ms}$, a $30.1 x$ performance increase over the 0.015 frames per second and $65568.20 \mathrm{~ms}$ of average latency of the Baseline system.

It is also interesting to compare the Baseline and MOPED in terms of system scalability. We are most interested in the scalability with respect to image resolution, number of objects in a scene and number of objects in the database. Our synthetic benchmarks allow for a controlled comparison of these different parameters without affecting the others.

The Rotation Benchmark contains images with a constant number of object instances at 5 different resolutions. Fig. 13.(left) shows that both MOPED and the Baseline system scale linearly in execution time with respect to image resolution, i.e. quadratically with respect to image width. To be more accurate, the implementation of ICE in both systems allows their performance to increase linearly with the number of feature clusters. The number of SIFT features and feature clusters also increase linearly with respect to image resolution in this Benchmark.

To test the scalability with respect to the number of objects in the database, images from the Rotation Benchmark are generated to have a fixed number of object instances and poses, and change the identity of the object instances from a minimum of 5 different objects to a maximum of 80 . The use of a database-wide feature matching technique (k-NN Per Database, Section 6.3.4) allows MOPED to perform almost constantly with respect to the number of objects in the database. The latency of the Baseline system, which performs independent feature matching per object, increases roughly linearly. Fig. 13 shows the latency of each system relative to their best scores, to see how latency increments when each of the parameters change. Therefore, it is important to note that the latency of MOPED and the Baseline are in different scales in Fig. 13, and one should only compare the relative differences when changing the image resolution and the size of the object database.

The number of objects in a scene is another factor that can greatly affect the performance of MOPED. The Zoom Benchmark aims to show a relatively constant number of image features (Fig. 14), despite being only 12 (large) objects visible at $280 \mathrm{~mm}$ and 160 (smaller) objects visible at $1050 \mathrm{~mm}$. It is interesting to notice that the required time is inversely proportional to the number of objects in the image, i.e. a small number of large objects are more demanding than large numbers of small objects. The explanation for this fact is that the smaller objects in this benchmark are more likely to fit in a single object prior in the first iteration of ICE. Clusters that converge after the first iteration of ICE (i.e. with no correspondences transferred to or from them) require very little processing time in the second iteration of ICE. On the other hand, bigger objects require more effort in the second iteration of ICE due to the cluster merging process. It is also worth noting that this experiment pushes the limits of our graphics card, causing an inevitable degradation in performance when the GPU memory limit is reached. In the $850 \mathrm{~mm}-1050 \mathrm{~mm}$ range, the number of SIFT features to compute is slightly lower than in 

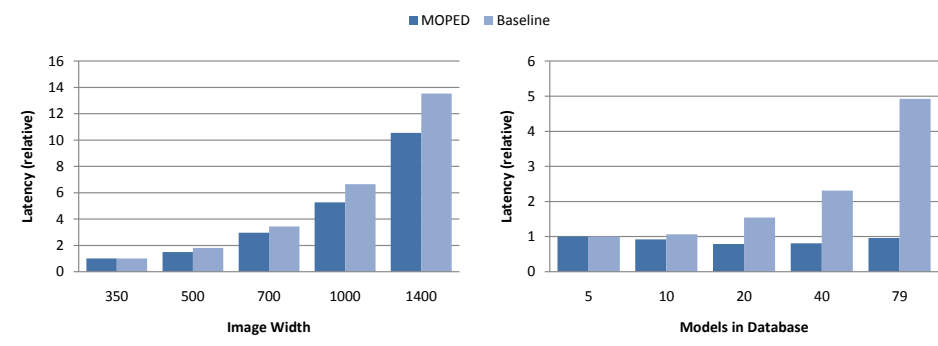

Figure 13: Scalability experiments in the Rotation Benchmark. (left) Latency with respect to image resolution. (right) Latency with respect to database size.
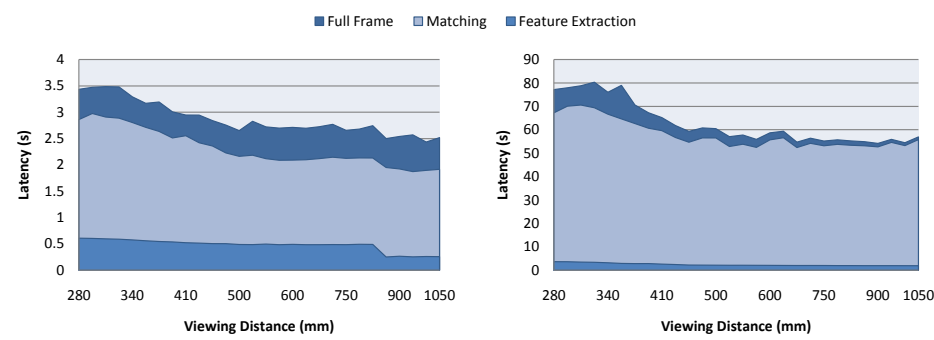

Figure 14: Scalability with respect to the number of objects in the scene in the Zoom Benchmark. The scale of the left chart is 22.5 times that of the right chart for better visibility. (left) Latency of MOPED. (right) Latency of the Baseline system.

the $280 \mathrm{~mm}-850 \mathrm{~mm}$ range. In the latter case, the memory limit of the GPU is reached, causing a two-fold increase in feature extraction latency when this happens. Despite this effect, the average latency for MOPED in the Zoom Benchmark is 2.4 seconds, compared to 65.5 seconds in the Baseline system.

\section{Recognition and Accuracy}

In this section, we evaluate the recognition rate, pose estimation accuracy and robustness of MOPED in the case of a singleview setup (MOPED-1V) and a three-view setup (MOPED$3 \mathrm{~V}$, shown in Fig. 16), and compare their results to other wellknown multi-view object recognition strategies.

We have conducted two sets of experiments to prove MOPED's suitability for robotic manipulation. The first set evaluates the accuracy of MOPED in estimating the position and orientation of a given object in a set of images. The second set of experiments evaluates the robustness of MOPED against modeling errors, which can greatly influence the accuracy of pose estimation. In all experiments, we estimate the full 6-DOF pose of objects, and no assumptions are made on their orientation or position. In all cases, we perform the image space clustering step with a Mean Shift radius of 100 pixels, and we use RANSAC with subsets of 5 correspondences to compute each hypothesis. The maximum number of RANSAC iterations is set to 500 in both Pose Estimation steps. In MOPED-3V, we enforce the requirement that a pose must be seen by at least two views, and that at least $50 \%$ of the points from the dif-

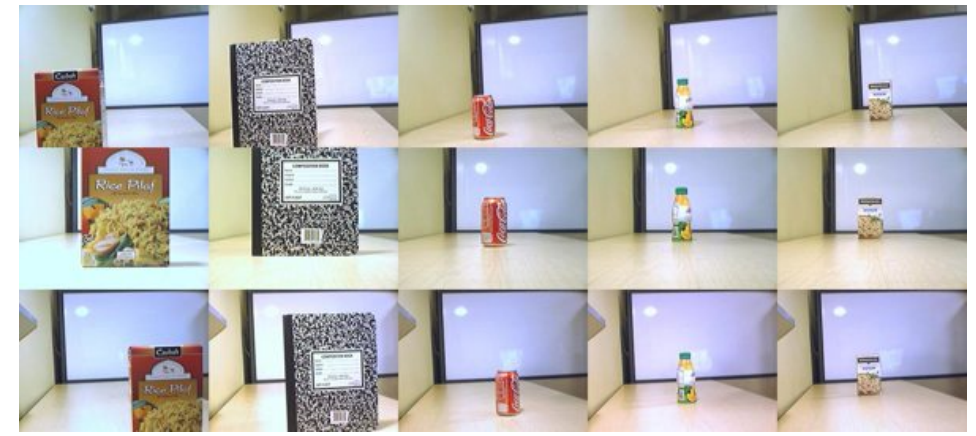

Figure 15: Examples scenes captured by our camera setup with Cam 1 (top), Cam 2 (middle), and Cam 3 (bottom). (Col 1) Rice box at $50 \mathrm{~cm}$. (Col 2) Notebook at $60 \mathrm{~cm}$. (Col 3) Coke can at $80 \mathrm{~cm}$. (Col 4) Juice bottle at $1 \mathrm{~m}$. (Col 5) Pasta box at $1.2 \mathrm{~m}$.

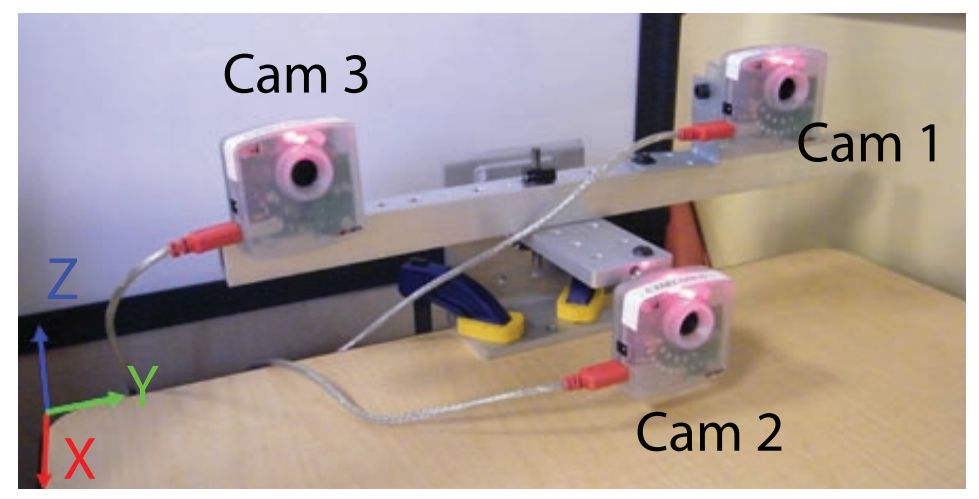

Figure 16: Three-camera setup used for accuracy tests with coordinate frame indicated on bottom left corner.

ferent hypotheses are consistent with the final pose. We add this requirement in order to prove that MOPED-3V takes full advantage of the multi-view geometry to improve its estimation results.

The experimental setup is a static three-camera setup with approximately $10 \mathrm{~cm}$ baseline between each two cameras (see Fig. 16). Both intrinsic and extrinsic parameters for each camera have been computed, considering camera 1 as the coordinate origin.

\subsection{Alternatives for multi-view recognition and estimation}

We consider two well-known techniques for object recognition and pose estimation in multiple simultaneous views. The techniques we consider are the Generalized Camera (Grossberg and Nayar, 2001) and the pose averaging (Viksten et al., 2006) techniques.

\subsubsection{Generalized Camera}

The Generalized Camera approach parameterizes a network of cameras as sets of rays that project from each camera center to each image pixel, thus expressing the network of cameras 
a single non-perspective camera with multiple projection centers and focal planes. Then, the camera pose is optimized in this generalized space by solving the resulting non-perspective PnP (i.e. nPnP) problem (Chen and Chang, 2004). While such an approach is perfectly valid, it might not be entirely feasible in real-time if the correspondence problem needs to be addressed as well, as the search space increases dramatically with each extra image added to the system. This process takes full advantage of the multi-view geometry constraints imposed by the camera setup, and its accuracy results can be considered a theoretical limit for multi-view model-based pose estimation. In our experiments, we implement this technique and use 1000 RANSAC iterations to robustly find correspondences in the generalized space.

\subsubsection{Pose averaging}

One of the simplest and most used alternatives for multi-view recognition is to combine multiple single-image algorithms via pose verification (Selinger and Nelson, 2001), robust averaging, or weighted voting (Viksten et al., 2006). These methods avoid the larger search space that may cause difficulties in the Generalized Image approach, but they fail to extract information from the multi-view geometry to provide a globally optimized pose estimate. In our experiments, we use the output of MOPED-1V and perform pose robust averaging using the Q-Scores of the MOPED-1V hypotheses as a weighting factor.

\subsection{Pose estimation accuracy}

In this set of experiments, we evaluate MOPED's accuracy over the range most useful in robotic manipulation. The threecamera setup was mounted and calibrated on a flat table (see Fig. 16). Our pose accuracy database is composed of five common household objects of various shapes and appearances. A set of 27 different positions and orientations for each object were gathered, with depths (i.e. distances from the central camera) ranging from $0.4 \mathrm{~m}$ to $1.2 \mathrm{~m}$ in $10 \mathrm{~cm}$ increments, lateral movements of up to $20 \mathrm{~cm}$ and out-of-plane rotations of up to 45 degrees. 10 images were taken with each camera at each position to account for possible image noise and artifacts, producing 810 images per object and a total of 4050 images. Some example images from this dataset are shown in Fig. 15.

It is important to mention that the choice of camera and lens can greatly affect pose estimation accuracy. The cameras we use in these experiments are low-cost cameras of $640 \times 480$ pixels with a $73^{\circ}$ field of view. The usage of a higher resolution image and a lens with a smaller field of view would greatly improve these results.

In all these experiments, the distance-normalized translation error refers to the absolute translation error divided by the distance with respect to the closest camera. Rotation error is measured as the quaternion angle $\alpha=2 \cos ^{-1}\left(q^{T} q_{g t}\right)$. The correct detection rate counts all pose hypotheses that lie within 5 $\mathrm{cm}$ and 10 degrees of the true pose. It is important to note that the correct detection, false positive and false negative rates do
Table 5: Average accuracy test. (1) MOPED-1V (2) Pose averaging. (3) MOPED-3V (4) Generalized Image.

\begin{tabular}{rcccc}
\hline & $(1)$ & $(2)$ & $(3)$ & $(4)$ \\
\hline TX error (cm) & 1.45 & 1.36 & 0.47 & $\mathbf{0 . 4 6}$ \\
TX error/dist. & $1.80 \%$ & $1.71 \%$ & $0.61 \%$ & $\mathbf{0 . 6 0} \%$ \\
Rot. error (deg) & 6.27 & 8.11 & 5.69 & $\mathbf{3 . 4 8}$ \\
Correct det. rate & $85.0 \%$ & $\mathbf{8 8 . 3} \%$ & $\mathbf{8 8 . 3} \%$ & $71.9 \%$ \\
False pos. rate & $2.78 \%$ & $\mathbf{0} \%$ & $\mathbf{0} \%$ & $\mathbf{0} \%$ \\
False neg. rate & $13.61 \%$ & $\mathbf{1 1 . 6 7} \%$ & $\mathbf{1 1 . 6 7} \%$ & $28.15 \%$ \\
Num iter./view & $\mathbf{9 6 . 7 1}$ & $\mathbf{9 6 . 7 1}$ & 98.69 & 259.05 \\
\hline
\end{tabular}

not necessarily need to add up to $100 \%$, because an algorithm might output a correct and an incorrect pose in the same image.

Table 5 compares the accuracy of MOPED-1V, robust pose averaging over MOPED-1V, MOPED-3V and the Generalized Image approach. MOPED-1V results show the average performance over the three cameras in our setup.

As we can see in Table 5, accuracy is increased threefold using MOPED-3V with respect to pose averaging, while requiring little overhead with respect to MOPED-1V. It is noteworthy that MOPED-3V and Generalized Image, considered a theoretical limit, perform very similarly in terms of accuracy, with a difference lower than $0.01 \%$. The low detection rate of the Generalized Image approach is due to its enormous computational cost, as it often exceeds the maximum number of iterations with no correct detection. The average number of iterations required to detect a single object with a Generalized Image approach is three times greater than MOPED, and its computational complexity grows exponentially with respect to the number of objects in a scene.

\subsection{Robustness against modeling noise}

In this set of experiments, we evaluate MOPED's robustness against modeling inaccuracies. Successful pose estimation in MOPED-1V is heavily dependent on a good model calibration, especially in terms of scaling, because depth is estimated entirely based on the scale of each model. Therefore, extreme care needs to be taken when generating models to set a proper scale, and we often require several tests before a new object model can be incorporated into the robot's object database. For example, a modeling error of $1 \mathrm{~mm}$ in a coke can (i.e. $1 \mathrm{~mm}$ larger than its real size), translates into a depth estimation error of up to $3 \mathrm{~cm}$ at a distance of $1 \mathrm{~m}$, large enough to cause problems to the robotic manipulator. On the other hand, having multiple views of the same object enables the use of further constraints in its pose. In MOPED-3V, an "implicit triangulation" takes place during the optimization, with the object drifting to its true position to minimize the global backprojection error imposed by the multi-view geometry, despite the larger error when MOPED-1V processes each view individually.

Table 6 and Table 7 showcase the effect of scaling errors during the object modeling stage. MOPED-3V outperforms ev- 


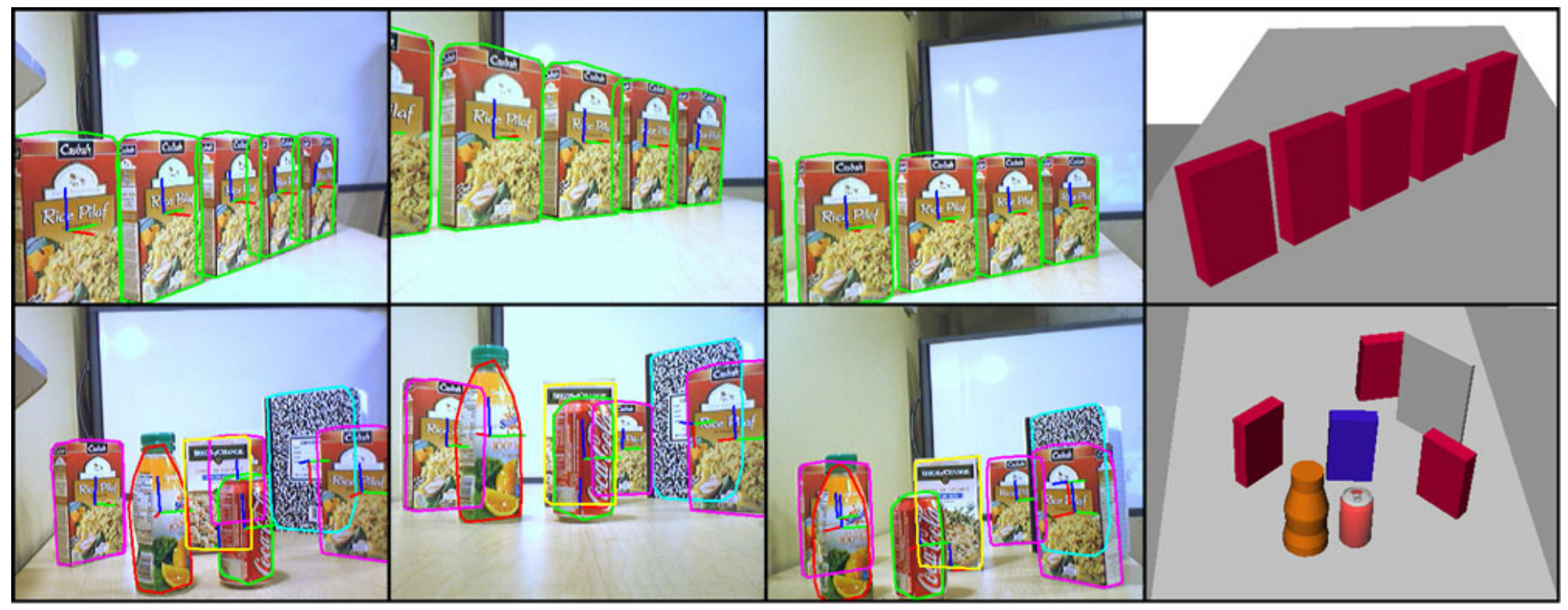

Figure 17: Performance of MOPED-3V in complex scenes. (Cols 1-3) depict the recognized poses overlaid on each image. (Col 4) shows a reconstruction of the given scenes in our virtual environment.

Table 6: Average distance-normalized translation error with varying model scale. See Table 5 for $(1),(2),(3),(4)$

\begin{tabular}{ccccc}
\hline Model scale & $(1)$ & $(2)$ & $(3)$ & $(4)$ \\
\hline 0.95 & $4.11 \%$ & $4.20 \%$ & $\mathbf{0 . 8 1} \%$ & $\mathbf{0 . 8 1} \%$ \\
0.97 & $2.56 \%$ & $2.65 \%$ & $0.68 \%$ & $\mathbf{0 . 6 2} \%$ \\
0.99 & $1.86 \%$ & $1.76 \%$ & $0.61 \%$ & $\mathbf{0 . 5 4} \%$ \\
1.01 & $2.12 \%$ & $1.95 \%$ & $0.74 \%$ & $\mathbf{0 . 6 9} \%$ \\
1.03 & $3.14 \%$ & $2.90 \%$ & $0.98 \%$ & $\mathbf{0 . 9 4} \%$ \\
1.05 & $4.72 \%$ & $4.43 \%$ & $1.29 \%$ & $\mathbf{1 . 1 8} \%$ \\
\hline
\end{tabular}

Table 7: Average Correct detection rate with varying model scale. See Table 5 for $(1),(2),(3),(4)$

\begin{tabular}{ccccc}
\hline Model scale & $(1)$ & $(2)$ & $(3)$ & $(4)$ \\
\hline 0.95 & $69.7 \%$ & $71.7 \%$ & $\mathbf{8 0 . 8} \%$ & $59.3 \%$ \\
0.97 & $82.2 \%$ & $85.0 \%$ & $\mathbf{8 5 . 8} \%$ & $66.7 \%$ \\
0.99 & $84.4 \%$ & $\mathbf{8 6 . 7} \%$ & $\mathbf{8 6 . 7} \%$ & $71.1 \%$ \\
1.01 & $84.2 \%$ & $\mathbf{8 8 . 3} \%$ & $\mathbf{8 8 . 3} \%$ & $70.4 \%$ \\
1.03 & $74.4 \%$ & $77.5 \%$ & $\mathbf{8 7 . 5 \%}$ & $65.2 \%$ \\
1.05 & $55.8 \%$ & $58.3 \%$ & $\mathbf{8 5 . 0} \%$ & $54.1 \%$ \\
\hline
\end{tabular}

ery other approach in terms of recognition rate, while achieving similar accuracy results than the Generalized Image approach. The Generalized Image approach suffers from a major performance drop when modeling errors appear, since it is often not able to find subsets of correspondences that are consistent enough to generate good hypotheses. MOPED-1V correctly finds object hypotheses in each image, but modeling noise causes a drop in the correct detection rate, as the estimated poses are often outside the $5 \mathrm{~cm}$ threshold. MOPED-3V, on the other hand, finds object hypotheses in each image, and then uses the inherent multi-view constraints to correctly estimate the final object poses.

\section{Conclusion}

We have presented and validated MOPED, an optimized framework for the recognition and registration of objects that addresses the problems of high scene complexity, scalability and latency that hamper object recognition systems when working in real-world scenes. The use of Iterative Clustering-Estimation (ICE) integrates single- and multi-view object recognition in an efficient, robust, and easy to parallelize manner. The Hypothesis Quality Score and Projection Clustering work together to minimize the number of false positives and to re-utilize all available information in the accurate pose estimation of true positives. The multiple architectural improvements in MOPED provide over 30x improvement in latency and throughput, allowing MOPED to perform in real-time robotic applications.

The different accuracy and recognition experiments we performed in this paper gives us a quantitative evaluation of MOPED's capabilities. However, the most stringent performance test of an object recognition system for manipulation is to actually integrate it in a robotic platform and use it to 
interact with people in real time. MOPED has been, for the past two years, an active part of HERB (Srinivasa et al., 2010), and the pose estimation outputs of MOPED have been used to grasp more than 2000 objects. In a controlled experiment, HERB and MOPED achieved a $91 \%$ grasping success rate using a single-image setup (MOPED-1V) and $98 \%$ success rate using a three-camera setup and MOPED-3V.

MOPED, however, is not without limitations. While we have addressed the issue of handling scenes with high complexity with minimal latency, the recognition performance of MOPED is ultimately tied to the ability of finding enough local features in a given object. If an object is not textured enough, too far away, or has large specular reflections on its surface, the feature extraction/matching steps might not find enough correspondences in the object to perform any kind of recognition. In our experience, we have found that a minimum of 8 to 10 correspondences are necessary to successfully recognize an object and estimate its pose. Hsiao et al. (2010) showed that the ability to generate more features in a scene can result in enormous boosts in recognition rate for objects with little texture. It would be interesting to evaluate the performance of such an algorithm integrated in MOPED.

An additional issue that often arises in the model-based object recognition literature is the model building stage. The model building stage we use in MOPED (described in Collet et al. (2009)), despite being mostly automatic, still requires a certain amount of human supervision, and we have to carefully scale our objects to achieve proper pose estimation from a single view. An important path to follow in the future is the use of object discovery techniques and multi-modal data to generate accurate models for MOPED. In particular, we are working on joint camera-laser discovery of objects to eliminate the scale uncertainty and obtain more robust object boundaries. The use of laser data (or other kind of 3D information, such as RGB$\mathrm{D}$ cameras) to improve the Clustering and Estimation steps of MOPED is another promising line of work to be investigated in the future.

\section{Appendix}

\section{A Pose estimation from point corre- spondences}

There are two main error metrics to recover the pose of a $3 \mathrm{D}$ model from point correspondences, the reprojection and backprojection errors. Both error functions perform equivalently when estimating object poses in Euclidean space, so one may choose either one. The reprojection error is usually preferred in the computer vision community because it is invariant to projective transformations, while the backprojection error is meaningless in projective space (Hartley and Sturm, 1994). In our particular case, working with calibrated cameras in an Euclidean space, we have chosen the backprojection error because it makes our framework more easily extensible to other types of multi-modal data, such as LASER point clouds or RGBD cameras, which we plan to incorporate in the near future. This section contains a brief derivation of the error functions we use in MOPED, both for the reprojection and backprojection errors.

\section{A.1 Reprojection error}

Consider a set of correspondences $\mathbf{C}_{m}$ in image $m$, where each correspondence $C_{j ; m}^{o}=\left(f_{j ; m}, F_{i ; o}\right)$. Assume the corresponding features in $C_{j ; m}^{o}$ have locations $p_{j ; m}$ in an image and $P_{i ; o}$ in an object model. For a given transformation $T$ and an image $m$ with extrinsic parameters $T_{m}{ }^{1}$, the sum of reprojection errors is defined by

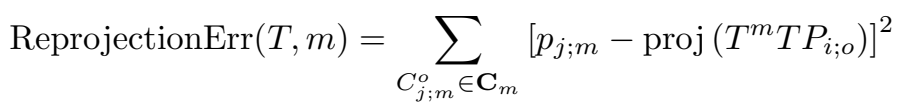

The optimal single hypothesis $h^{*}$ for a given set of correspondences $\mathbf{C}$ is one that minimizes the sum of reprojection errors of the correspondences across all images. The pose $T_{h}^{*}$ for $h^{*}$ is then defined as:

$$
T_{h}^{*}=\underset{T}{\arg \min } \sum_{m=1}^{M} \operatorname{Reprojection} \operatorname{Err}(T, m)
$$

\section{A.2 Backprojection Error}

Alternatively, one can define an analogous optimization in terms of the backprojection error, by tracing the line $L_{j ; m}$ from the camera center to each $2 \mathrm{D}$ point $p_{j ; m}$, and computing the distance from $L_{j ; m}$ to the corresponding $3 \mathrm{D}$ point $P_{i ; o}$. We parameterize a line as $L=(c, v)$, where $v$ is a unit vector indicating the line direction and $c$ is an arbitrary point on that line, e.g. the camera center. Using projective geometry, we obtain

$$
\bar{v}_{j ; m}=\frac{K_{m}^{-1} p_{j ; m}}{\left\|K_{m}^{-1} p_{j ; m}\right\|}
$$

where $K_{m}$ is a $3 \times 3$ intrinsic camera matrix for image $m$. Each line $L_{j ; m}$ in the world reference frame is then given by

$$
v_{j ; m}=\left(R_{m}\right)^{T} \bar{v}_{j ; m} \quad c_{j ; m}=-\left(R_{m}\right)^{T} t_{m}
$$

The distance between a point $P_{i ; o}$ and $L_{j ; m}$ is given by

$$
d\left(P_{i ; o}, L_{j ; m}\right)=\left\|\left(\mathcal{I}_{3 \times 3}-v_{j ; m} v_{j ; m}{ }^{T}\right)\left(P_{i ; o}-c_{j ; m}\right)\right\|
$$

The analogous equation to Eq. (19) that minimizes the sum of backprojection errors of a set of correspondences $\mathbf{C}$ with $C_{j}=\left(P_{i ; o}, L_{j ; m}\right)$ is given by

$$
T_{h}^{*}=\arg \min _{T} \sum_{i=1}^{M} \sum_{C_{j} \in \mathbf{C}}\left[d\left(T^{m} T P_{i ; o}, L_{j ; m}\right)\right]^{2}
$$

Additionally, we found it useful to constrain the objects to lie in front of the cameras. Given that $v_{j ; m}$ are vectors

\footnotetext{
${ }^{1}$ Reminder: in tensor notation, $T^{m}=\left(T_{m}\right)^{-1}$
} 
from the camera center pointing towards the image plane, $v_{j ; m}^{T}\left(P_{i ; o}-c_{j ; m}\right)>0$ for all points $P_{i ; o}$ in front of camera $m$. We incorporate this constraint as a regularizer (with weight $\xi>0)$ in the minimization

$$
\begin{aligned}
& T_{h}^{*}=\underset{T}{\arg \min } \sum_{i=1}^{M} \sum_{C_{j} \in \mathbf{C}} \\
& {\left[d\left(T^{m} T P_{i ; o}, L_{j ; m}\right)+\xi\left(1-v_{j ; m}{ }^{T} \frac{\left(P_{i ; o}-c_{j ; m}\right)}{\left\|P_{i ; o}-c_{j ; m}\right\|}\right)\right]^{2}(24}
\end{aligned}
$$

\section{Acknowledgments}

This material is based upon work partially supported by the National Science Foundation under Grant No. EEC-0540865. Alvaro Collet is partially supported by Caja Madrid fellowship. Special thanks to Martial Hebert, Chris Atkeson and members of the Personal Robotics Lab at Intel Pittsburgh for insightful comments and discussions.

\section{References}

AMD. ATI Radeon HD 5970 Graphics Feature Summary, 2010. URL http://www.amd.com/us/products/ desktop/graphics/ati-radeon-hd-5000/hd-5970/ Pages/ati-radeon-hd-5970-specifications . aspx.

Alexandr Andoni and Piotr Indyk. Near-optimal hashing algorithms for approximate nearest neighbor in high dimensions. In International Symposium on Foundations of Computer Science, pages 459-468. IEEE, 2006.

Sunil Arya, David M. Mount, Nathan S. Netanyahu, Ruth Silverman, and Angela Y. Wu. An optimal algorithm for approximate nearest neighbor searching fixed dimensions. Journal of the ACM, 45(6), 1998.

Herbert Bay, Andreas Ess, Tinne Tuytelaars, and Luc Van Gool. Speeded-Up Robust Features (SURF). Computer Vision and Image Understanding, 110(3):346-359, 2008.

K.S. Chatha and R. Vemuri. Hardware-software partitioning and pipelined scheduling of transformative applications. IEEE Transactions on Very Large Scale Integration (VLSI) Systems, 10(3):193-208, June 2002.

Chu-Song Chen and Wen-Yan Chang. On Pose Recovery For Generalized Visual Sensors. IEEE Transactions on Pattern Analysis and Machine Intelligence, 26(7):848-61, July 2004.

Yizong Cheng. Mean Shift, Mode Seeking, and Clustering. IEEE Transactions on Pattern Analysis and Machine Intelligence, 17(8):790, 1995.

Alvaro Collet and Siddhartha S. Srinivasa. Efficient Multi-View Object Recognition and Full Pose Estimation. In IEEE International Conference on Robotics and Automation, Anchorage, May 2010. IEEE.

Alvaro Collet, Dmitry Berenson, Siddhartha S. Srinivasa, and Dave Ferguson. Object recognition and full pose registration from a single image for robotic manipulation. In IEEE International Conference on Robotics and Automation, pages 48-55, Kobe, May 2009. IEEE.

G. Conte, S. Tommesani, and F. Zanichelli. The Long And Winding Road to High-Performance Image Processing with MMX/SSE. In IEEE International Workshop on Computer Architectures for Machine Perception, page 302. IEEE, 2000.

Nico Cornelis and Luc Van Gool. Fast scale invariant feature detection and matching on programmable graphics hardware. In IEEE Conference on Computer Vision and Pattern Recognition Workshops, 2008.

Daniel F. Dementhon and Larry S. Davis. Model-based object pose in 25 lines of code. International Journal of Computer Vision, 15(1-2):123-141, June 1995.

A. P. Dempster, N. M. Laird, and D. B. Rubin. Maximum likelihood from incomplete data via the EM algorithm. Journal of the Royal Statistical Society, Series B, 39(1):1-38, 1977. 
Piotr Dollár and Vincent Rabaud. Piotr Dollar's Image \& Video Toolbox for Matlab, 2010. URL http://vision.ucsd.edu/ $\sim\{\}$ pdollar/toolbox/doc/index .html.

T.J. Dysart, B.J. Moore, L. Schaelicke, and P.M. Kogge. Cache implications of aggressively pipelined high performance microprocessors. In IEEE International Symposium on Performance Analysis of Systems and Software, pages 123-132, 2004.

Martin Fischler and Robert Bolles. Random sample consensus: a paradigm for model fitting with applications to image analysis and automated cartography. Communications of the ACM, 24(6), 1981.

W. Eric L. Grimson. Object Recognition by Computer. MIT Press, 1991. ISBN 0-262-07130-4.

Michael D. Grossberg and Shree K. Nayar. A general imaging model and a method for finding its parameters. In IEEE International Conference on Computer Vision, pages 108115. IEEE, 2001.

Richard Hartley and Peter Sturm. Triangulation, 1994.

Edward Hsiao, Alvaro Collet, and Martial Hebert. Making specific features less discriminative to improve point-based $3 \mathrm{D}$ object recognition. In IEEE Conference on Computer Vision and Pattern Recognition, 2010.

Peter J. Huber. Robust Statistics. Wiley, 1981.

Intel Corp. Core i7 Performance, 2010. URL http://www . intel.com/support/processors/sb/cs-023143.htm.

David S. Johnson. Fast algorithms for bin packing. Journal of Computer and System Sciences, 8(3):42, 1974.

Vincent Lepetit and Pascal Fua. Monocular Model-Based 3D Tracking of Rigid Objects: A Survey. Foundations and Trends in Computer Graphics and Vision, 1(1):1-89, 2005.

Vincent Lepetit, Francesc Moreno-Noguer, and Pascal Fua. EPnP: An Accurate $\mathrm{O}(\mathrm{n})$ Solution to the PnP Problem. International Journal of Computer Vision, 81(2):155-166, February 2008.

Manolis Lourakis. LEVMAR: A Levenberg-Marquardt C++ Implementation, 2010. URL http://www.ics.forth.gr/ $\sim\{\}$ lourakis/levmar.

David Lowe. Three-dimensional object recognition from single two-dimensional images. Artificial Intelligence, 31(3):355395, March 1987.

David Lowe. Distinctive Image Features from Scale-Invariant Keypoints. International Journal of Computer Vision, 60(2): 91-110, 2004.

Donald W Marquardt. An Algorithm for Least-Squares Estimation of Nonlinear Parameters. SIAM Journal on Applied Mathematics, 11(2):431-441, 1963.
Manuel Martinez, Alvaro Collet, and Siddhartha S. Srinivasa. MOPED: A Scalable and low Latency Object Recognition and Pose Estimation System. In IEEE International Conference on Robotics and Automation, Anchorage, 2010. IEEE.

Krystian Mikolajczyk and Cordelia Schmid. A Performance Evaluation of Local Descriptors. IEEE Transactions on Pattern Analysis and Machine Intelligence, 27(10), 2005.

Marius Muja and David Lowe. Fast approximate nearest neighbors with automatic algorithm configuration. In International Conference on Computer Vision Theory and Applications, pages 331-340, 2009.

Clark F. Olson. Efficient Pose Clustering Using a Randomized Algorithm. International Journal of Computer Vision, 23(2): $131,1997$.

Mustafa Ozuysal, Michael Calonder, Vincent Lepetit, and Pascal Fua. Fast keypoint recognition using random ferns. IEEE Transactions on Pattern Analysis and Machine Intelligence, 32(3):448-61, March 2010.

Richard A. Redner and Homer F. Walker. Mixture Densities, Maximum Likelihood and the EM Algorithm. SIAM Review, 26(2):195-239, 1984.

Gerald Schweighofer and Axel Pinz. Robust pose estimation from a planar target. IEEE Transactions on Pattern Analysis and Machine Intelligence, 28(12):2024-30, 2006.

Andrea Selinger and Randal C. Nelson. Appearance-based object recognition using multiple views. In IEEE Conference on Computer Vision and Pattern Recognition, pages 905-911. IEEE, 2001.

Chanop Silpa-Anan and Richard Hartley. Optimised KD-trees for fast image descriptor matching. In IEEE Conference on Computer Vision and Pattern Recognition, pages 1-8. IEEE, 2008 .

Siddhartha S. Srinivasa, Dave Ferguson, Casey J. Helfrich, Dmitry Berenson, Alvaro Collet, Rosen Diankov, Garratt Gallagher, Geoffrey Hollinger, James Kuffner, and Michael Vande Weghe. HERB: a home exploring robotic butler. Autonomous Robots, 28(1):5-20, 2010.

Richard Szeliski and Sing Bing Kang. Recovering 3D shape and motion from image streams using nonlinear least squares. Journal of Visual Communication and Image Representation, 5(1):10-28, March 1994.

J. Van Weveren. From Quaternion to Matrix and Back, 2005.

Fredrik Viksten, Robert Soderberg, Klas Nordberg, and Christian Perwass. Increasing pose estimation performance using multi-cue integration. In IEEE International Conference on Robotics and Automation, pages 3760-3767. IEEE, 2006. 
Barry Wilkinson and Michael Allen. Parallel Programming: Techniques and Applications Using Networked Workstations and Parallel Computers, chapter 3. Prentice Hall, 2 edition, 2004.

WillowGarage. The Personal Robot Project, 2008. URL http: //www.willowgarage.com.

Changchang Wu. SiftGPU: A GPU Implementation of Scale Invariant Feature Transform (SIFT), 2007. URL http:// cs. unc. edu/ \{\} ccwu/siftgpu.

Zhengyou Zhang. Parameter Estimation Techniques: A Tutorial with Application to Conic Fitting. Image and Vision Computing, 15(1):59-76, January 1997. 